\title{
Development of scaling criteria for steam flooding EOR process
}

\author{
Arifur Rahman $^{1,2}$ [ Salim Ahmed ${ }^{1} \cdot$ M. Enamul Hossain ${ }^{3} \cdot$ Fatema Akter Happy $^{1}$
}

Received: 27 February 2020 / Accepted: 11 May 2020 / Published online: 30 May 2020

(c) The Author(s) 2020

\begin{abstract}
The development of new scaling criteria for steam flooding process is presented in this paper. The mathematical development is done by using modified Darcy's law, constitutive relationships, constraints, and the initial and boundary conditions. Dimensional and inspectional analyses are used to develop sets of dimensionless groups by incorporating rock and fluid memory concept. The variety of scaling criteria and their comparative advantages and limitations are discussed. Currently available scaling criteria development for steam flooding processes used the same fluid, same porous media in model and prototype. However, it requires a high-pressure model with different porous media, which causes difficulties in scaling properties, and therefore, largely depends on pressure and the porous media itself. In this paper, different methods are presented which permit scaling of all properties dependent on pressure or temperature by relaxing the requirements of geometric similarity. A set of relaxed scaling criteria is determined to satisfy a major mechanism. A comparative study of different approaches and their relative merits and demerits are discussed. Approach 2 (same fluids, same pressure drop, same porous medium, and geometric similarity) seems to be the most appropriate for the steam flooding process; however, gravitational forces cannot be scaled properly with this approach. Approach 3 (same fluids, same pressure drop, same porous media, and relaxed geometric similarity) is suitable for this process if the effect of transverse dispersion is considered negligible. Finally, a table is developed which can act as a guideline to select an appropriate approach that best scales a major mechanism for a specific steam flooding recovery process.
\end{abstract}

Keywords Steam flooding $\cdot$ Dimensionless numbers $\cdot$ Dimensional analysis $\cdot$ Inspectional analysis $\cdot$ Memory concept

$\begin{array}{ll}\text { List of symbols } \\ A & \text { Area }\left(\mathrm{m}^{2}\right) \\ L & \text { Reservoir length (m) } \\ W & \text { Reservoir width }(\mathrm{m}) \\ H & \text { Reservoir thickness }(\mathrm{m}) \\ c & \text { Compressibility }(\mathrm{m} \mathrm{s} / \mathrm{kg}) \\ \phi & \text { Porosity (fraction) } \\ k & \text { Permeability }\left(\mathrm{m}^{2}\right) \\ k_{\mathrm{r}} & \text { Relative permeability }\left(\mathrm{m}^{2} / \mathrm{m}^{2}\right) \\ P & \text { Pressure }\left(\mathrm{kg} / \mathrm{m}^{2}\right) \\ q_{\mathrm{i}} & \text { Injection rate }\left(\mathrm{m}^{3} / \mathrm{s}\right) \\ q_{\text {ia }} & \text { Injection rate of additive }\left(\mathrm{m}^{3} / \mathrm{s}\right) \\ q_{\text {prod }} & \text { Production rate }\left(\mathrm{m}^{3} / \mathrm{s}\right)\end{array}$

Arifur Rahman

ar0727@mun.ca

1 Department of Process Engineering, Memorial University of Newfoundland, St. John's, Canada

2 Department of Petroleum and Mining Engineering, Shahjalal University of Science and Technology, Sylhet, Bangladesh

3 Department of Petroleum and Energy Engineering, The American University in Cairo, Cairo, Egypt

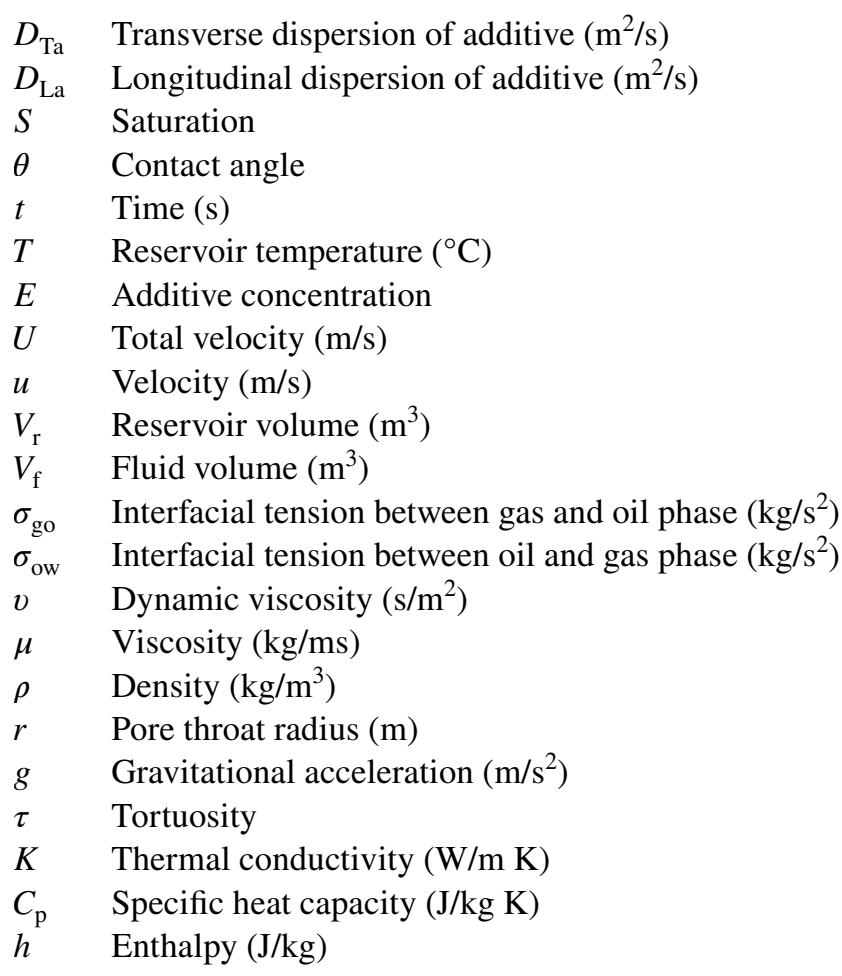

$D_{\mathrm{Ta}} \quad$ Transverse dispersion of additive $\left(\mathrm{m}^{2} / \mathrm{s}\right)$

$D_{\mathrm{La}} \quad$ Longitudinal dispersion of additive $\left(\mathrm{m}^{2} / \mathrm{s}\right)$

$S \quad$ Saturation

$\theta \quad$ Contact angle

$t \quad$ Time (s)

$T \quad$ Reservoir temperature $\left({ }^{\circ} \mathrm{C}\right)$

E Additive concentration

$U$ Total velocity $(\mathrm{m} / \mathrm{s})$

$u \quad$ Velocity $(\mathrm{m} / \mathrm{s})$

$V_{\mathrm{r}} \quad$ Reservoir volume $\left(\mathrm{m}^{3}\right)$

$V_{\mathrm{f}} \quad$ Fluid volume $\left(\mathrm{m}^{3}\right)$

$\sigma_{\text {go }}$ Interfacial tension between gas and oil phase $\left(\mathrm{kg} / \mathrm{s}^{2}\right)$

$\sigma_{\text {ow }}$ Interfacial tension between oil and gas phase $\left(\mathrm{kg} / \mathrm{s}^{2}\right)$

$v \quad$ Dynamic viscosity $\left(\mathrm{s} / \mathrm{m}^{2}\right)$

$\mu \quad$ Viscosity $(\mathrm{kg} / \mathrm{ms})$

$\rho \quad$ Density $\left(\mathrm{kg} / \mathrm{m}^{3}\right)$

$r \quad$ Pore throat radius (m)

$g$ Gravitational acceleration $\left(\mathrm{m} / \mathrm{s}^{2}\right)$

$\tau \quad$ Tortuosity

$K$ Thermal conductivity (W/m K)

$C_{\mathrm{p}} \quad$ Specific heat capacity $(\mathrm{J} / \mathrm{kg} \mathrm{K})$

$h$ Enthalpy $(\mathrm{J} / \mathrm{kg})$ 
$L_{\mathrm{v}} \quad$ Latent heat $(\mathrm{J} / \mathrm{kg})$

$\xi \quad$ Dummy variable for time (s)

\section{Subscript}

$f \quad$ Fluid

$o \quad$ Oil phase

$w \quad$ Water phase

$g \quad$ Gaseous phase

$i \quad$ Initial

$r \quad$ Rock or reservoir

$t \quad$ Total

\section{Introduction}

Dimensionally scaled model studies have been applied to engineering problems for many years, especially in the field of heat transfer and fluid flow with structural design (Leverett et al. 1942; Mattax and Kyte 1962; Bridgman 1931; Langhaar 1951; Murphy 1950). A similar approach used in the field of petroleum engineering is relatively new; however, its application is increasing day by day (Leverett et al. 1942; Bobek and Bail 1961; Mattax and Kyte 1962; Carpenter et al. 1962; Graham and Richardson 1959; Rapoport 1955; Craig et al. 1955, 1957; Rapoport and Leas 1953; Engelberts and Klinkenberg 1951; Seve and Pottier 1963; Geertsma et al. 1956; Van Meurs 1956; Henley et al. 1961; Jadhawar 2010; Rahman 2018; Rahman et al. 2017; Hossain 2017). It can reproduce the behaviour of one scale process to another scale. Many researchers had demonstrated the importance of scaling criteria development in many ways (Kimber et al. 1988; Shook et al. 1992; Novakovic 2002). However, the importance of scaling criteria development can be demonstrated for new processes whose mechanisms are not known to us. Moreover, the mathematical description of these processes is difficult to formulate. Many small-scale models had been developed for multiphase flow behaviour considering reservoir as either isothermal or non-isothermal process.

Laboratory models had been used for many years to extrapolate the behaviour of thermal recovery operations. It is unfortunate that the scaling criteria development of the thermal recovery processes is difficult to formulate since a lot of factors are involved in such recovery processes. Heat transfer and thermal effects of fluid and rock properties, as well as gravity, capillary, and viscous forces, should be considered for the thermal recovery process. The steam flooding technique is usually used for heavy or viscous oil reservoirs.

The laboratory steam flooding process had been evolved during the last two decades from qualitative observations to the complicated scaled model. Researchers investigated many procedures for scaling the steam flooding process. Farouq Ali and Redford (1977) reviewed the approach used by previous investigators. The different approaches show a different degree of complexity. Stegemeier et al. (1980) developed the most widely used scaling technique for lowpressure models. On the other hand, Pujol and Boberg (1972) investigated the scaling technique for high-pressure models. In a high-pressure model, the same fluids will be used in the model and prototype. Low-pressure models are easy to generate and operate where different fluids are used in the model and prototype. Huygen (1976), and Huygen and Lowry (1983) developed a high-pressure model by considering heat flow in crudes and crushed sandstones. The authors investigated the effect of oil viscosity, distillation, and initial oil saturation on oil recovery. Pursley (1974) developed the high-pressure scaled model to study the effect of bottom water, gas cap, heterogeneities, and steam quality on reservoir response. Ehrlich et al. (1974) developed a scaled model by using Pujol and Boberg's scaling laws for Wabasca, Alberta, heavy oil. An intermediate pressure model had been constructed by Lo (1977) for 1/12 of a seven-spot pattern. Singhal (1980) developed a scaled model depending on steam quality, including the enthalpy of vapour to liquid water. The author simulated Lloydminster type oil sand by material and energy balance equation.

This study is focused on deriving scaling criteria using inspectional and dimensional analysis. A relaxed set of criteria is determined without significantly changing the process parameters. The geometric similarity is relaxed to satisfy other requirements for the steam flooding process. Five different sets of scaling criteria approaches are developed and their comparative study performed to discover which approach is most suitable for the steam flooding process.

\section{Development of scaling criteria}

The application of scaling law is dependent on the concept of dimensional similarity. A correctly scaled model requires physical, dynamic, and geometric similarity at each point between the model and prototype (Poettmann et al. 1974). However, all physical and geometric variables are essentially proportional to a perfectly scaled model at any time and point. Moreover, governing equations and their initial and boundary conditions are also satisfied with the similarity criteria regarding dimensionless parameters. There are two standard procedures for deriving scaling criteria for any system. These are inspectional analysis (Ruark 1935; Birkhoff 1950; Bear 1972; Shook et al. 1992, Novakovic 2002) and dimensional analysis (Buckingham 1914; Bridgman 1931; Langhaar 1951; Focken 1953; Nielsen and Tek 1963; Sonin 2001). 


\section{Dimensional analysis}

The dimensional analysis is a technique to form any dimensionless group using two or more variables. The impacts of different variables are then studied in a group rather than individually in the group. The dimensional analysis method combines variables that affect a process or system into fundamental dimensionless numbers. Through this process, functions and experiments are simplified by the combination of the various variables. When dimensionless groups are derived, it lumps together the numerous variables which affect a process. It would be cumbersome to run a series of experiments to define how the parameters affect each other. Two methods used for dimensional analysis include:

1. The Rayleigh method and

2. The Buckingham PI Theorem

The choice of the above methods in the derivation of the dimensionless numbers depends largely on the number of variables involved in describing the phenomena. The Rayleigh's method is utilized for processes involving few variables. For the processes or system involving many variables, the Buckingham PI theorem is used.

\section{Rayleigh's method}

The Rayleigh's method is summarized in Fig. 1.

Here $k$ is a dimensionless constant; $a, b, c, d$ variables; and $m, n, o, \mathrm{p}$ arbitrary exponents.

\section{Buckingham Pi theorem}

The procedure for the Buckingham Pi theorem is given below:

1. List all variables (the independent variables)

2. Express each variable in basic dimensions

3. Determine the required number of PI terms

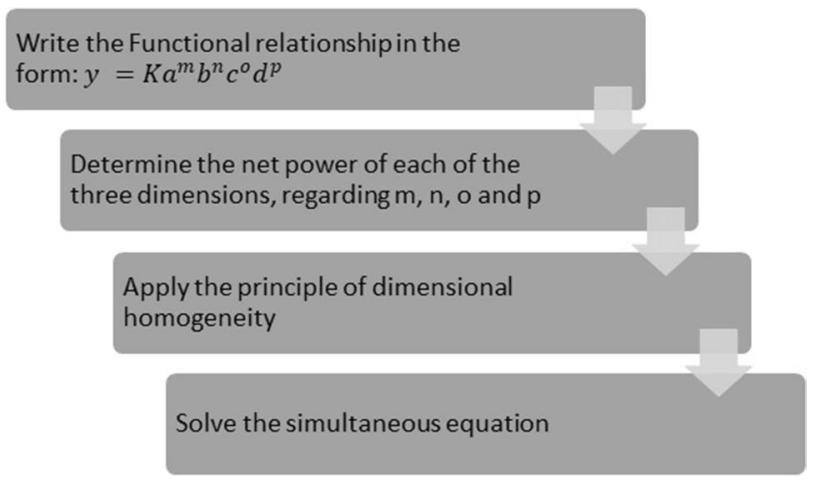

Fig. 1 Rayleigh's method

(1) $\pi s=k-r$

$K=$ number of physical relationships and $r=$ number of reference dimensions required to describe the variables

4. Select $r$ repeating variables by

(i) Avoid dependent variables.

(ii) Ensure that each is dimensionally independent and cannot be combined to form dimensionless numbers.

5. Form the Pi terms by multiplying the non-repeating variables by repeating variables to get dimensional numbers.

(i) Repeating variables can be raised to any power.

(ii) Non-repeating variables are raised to the power of 1 .

\section{Mathematical formulation using $\pi$-theorem}

Leverett et al. (1942) used dimensionless groups for the investigation of reservoir behaviour by adopting dimensional analysis.

If any variable $p_{1}$ depends upon the independent variables, $p_{2}, p_{3}, p_{4} \ldots p_{n}$ then we may write:

$p_{1}=f\left(p_{2}, p_{3}, p_{4} \ldots p_{n}\right)$

where $p_{1}$ is the dependent parameter, and $p_{2}, p_{3}, p_{4} \ldots p_{n}$ are $(n-1)$ numbers of the independent parameter. Since there exists a mathematical equilibrium between the dependent and the independent variables, they may be grouped into another functional relationship equal to zero:

$g\left(p_{2}, p_{3}, p_{4} \ldots p_{n}\right)=0$.

where $g$ is an unspecified function, making the transformations to dimensionless form simple and straightforward. Several steps have to be taken to decide the required numbers of dimensionless groups. The primary step is to determine the total number of primary dimensions " $n$ " which involve the physical processes. Then determine the number of repeating parameters assigned as " $m$." To find out the value of " $m$," it is required to determine the rank of the resulting dimensional matrix:

$m=\left[\begin{array}{ccc}a 11 & \cdots & a n 1 \\ \vdots & \ddots & \vdots \\ a 1 r & \cdots & \text { anr }\end{array}\right]$

According to Buckingham, the dimensionless form of the equation should satisfy the following functional form:

$X\left(\left(\pi_{1}, \pi_{2}, \pi_{3}, \pi_{4} \ldots \pi_{n-m}\right)\right)=0$.

Rayleigh's method 
where $n-m$ denotes the minimum number of independent dimensionless groups which affect the physical process and is required to denote the dimensions of all parameter $p_{1}, p_{2}, p_{3} \ldots p_{n}$.

Table 1 shows the similarity group developed from the dimensional analysis. The pertinent variables are selected depending on the processes, and the Buckingham $\pi$-theorem is used to develop these dimensionless numbers. There are a few new groups that have been introduced through this approach, which is not found by inspectional analysis. It happened because some of the variables are not considered in the formulation of governing equations. The new groups formed by dimensional analysis had an insignificant or negligible effect on this specific process. So, their scaling requirements are relaxed.

\section{Inspectional analysis}

The inspectional analysis involves the formulation of the governing partial differential equations and initial and boundary conditions to derive dimensionless groups. Constitutive relationships and constraints were also formulated to derive these dimensionless groups. Derived dimensionless groups are written in terms of dimensionless variables with their reference quantities. Some of the dimensionless groups are eliminated, which have little or no effects on the specific process. In the inspectional analysis, the mathematical equation of a given problem is reduced to non-dimensional units of space, time, and mass. The process, like the dimensional analysis approach, generates sets of non-dimensional numbers appearing as coefficients in the governing equations.

The process involves:

1. To change the physical equation to non-dimensional equations. Non-dimensional equations are obtained by dividing each term in the equation by variables or constants whose products have the same dimensions.

2. Generation of non-dimensional parameters: In the process of making equations, non-dimensional parameters can be generated. The dimensionless parameters include sets of dimensional variables, non-dimensional variables, and dimensional constants in the problem.

The process involves in three-phase flows (i.e., oleic, aqueous, gaseous). The mass and energy balance takes place among distinct phases and additives. Modified Darcy's law and Fourier's law are used in deriving the dimensionless groups.
Table 1 Dimensionless group from dimensional analysis

\begin{tabular}{|c|c|c|c|c|}
\hline$\pi_{1}=\frac{A}{L^{2}}$ & $\pi_{13}=\frac{P_{i}}{\rho_{o} L T C_{p f}}$ & $\pi_{25}=s_{o}$ & $\pi_{37}=\frac{\sqrt{T C_{p f}}}{L^{-3 / 2}} v$ & $\pi_{49}=\frac{k_{w}}{\rho_{o} L^{5 / 2} T^{1 / 2} C_{p f}^{5 / 2}}$ \\
\hline$\pi_{2}=\frac{w^{2}}{L^{2}}$ & $\pi_{14}=\frac{P_{o}}{\rho_{o} L T C_{p f}}$ & $\pi_{26}=s_{w}$ & $\pi_{38}=\frac{T \mu_{o}}{\rho_{o} L^{2}}$ & $\pi_{50}=\frac{k_{o}}{\rho_{o} L^{5 / 2} T^{1 / 2} C_{p f}^{5 / 2}}$ \\
\hline$\pi_{3}=\frac{h^{2}}{L^{2}}$ & $\pi_{15}=\frac{P_{w}}{\rho_{o} L T C_{p f}}$ & $\pi_{27}=\theta$ & $\pi_{39}=\frac{T \mu_{w}}{\rho_{o} L^{2}}$ & $\pi_{51}=\frac{k_{r}}{\rho_{o} L^{5 / 2} T^{1 / 2} C_{p f}^{5 / 2}}$ \\
\hline$\pi_{4}=\frac{\rho_{o} L^{4} c_{r}}{T C_{p f}}$ & $\pi_{16}=\frac{P_{g}}{\rho_{o} L T C_{p f}}$ & $\pi_{28}=\frac{\sqrt{T} t}{\sqrt{A C_{p f}}}$ & $\pi_{40}=\frac{T \mu_{g}}{\rho_{o} L^{2}}$ & $\pi_{52}=\frac{C_{p g}}{C_{p f}}$ \\
\hline$\pi_{5}=\frac{\rho_{o} L^{4} c_{f}}{T C_{p f}}$ & $\pi_{17}=\frac{P_{c g o}}{\rho_{o} L T C_{p f}}$ & $\pi_{29}=\frac{U}{\sqrt{L T C_{p f}}}$ & $\pi_{41}=\frac{\rho_{g}}{\rho_{o}}$ & $\pi_{53}=\frac{C_{p r}}{C_{p f}}$ \\
\hline$\pi_{6}=\frac{\rho_{o} L^{4} c_{0}}{T C_{p f}}$ & $\pi_{18}=\frac{P_{c o w}}{\rho_{o} L T C_{p f}}$ & $\pi_{30}=\frac{u_{o}}{\sqrt{L T C_{p f}}}$ & $\pi_{42}=\frac{\rho_{w}}{\rho_{o}}$ & $\pi_{54}=\frac{C_{p w}}{C_{p f}}$ \\
\hline$\pi_{7}=\frac{\rho_{o} L^{4} c_{g}}{T C_{p f}}$ & $\pi_{19}=\frac{q_{i}}{\sqrt{T C_{p f} L} 5 / 2}$ & $\pi_{31}=\frac{u_{w}}{\sqrt{L T C_{p f}}}$ & $\pi_{43}=\frac{\nabla \rho}{\rho_{o}}$ & $\pi_{55}=\frac{h_{w}}{L T C_{p f}}$ \\
\hline$\pi_{8}=\frac{\rho_{o} L^{4} c_{w}}{T C_{p f}}$ & $\pi_{20}=\frac{q_{i a}}{\sqrt{T C_{p f}} L / 2}$ & $\pi_{32}=\frac{u_{g}}{\sqrt{L T C_{p f}}}$ & $\pi_{44}=\frac{r^{2}}{L^{2}}$ & $\pi_{56}=\frac{h_{o}}{L T C_{p f}}$ \\
\hline$\pi_{9}=\frac{\rho_{o} L^{4} c_{t}}{T C_{p f}}$ & $\pi_{21}=\frac{q_{p r o d}}{\sqrt{T C_{p f} L} 5 / 2}$ & $\pi_{33}=\frac{V_{r}}{L^{3}}$ & $\pi_{45}=\frac{g}{T C_{p f}}$ & $\pi_{57}=\frac{h_{g}}{L T C_{p f}}$ \\
\hline$\pi_{10}=\phi$ & $\pi_{22}=\frac{D_{T a}}{\sqrt{T C_{p f} L}{ }^{3 / 2}}$ & $\pi_{34}=\frac{V_{f}}{L^{3}}$ & $\pi_{46}=\tau$ & $\pi_{58}=\frac{h_{r}}{L T C_{p f}}$ \\
\hline$\pi_{11}=\frac{k^{2}}{L^{2}}$ & $\pi_{23}=\frac{D_{L a}}{\sqrt{T C_{p f} L}{ }^{3 / 2}}$ & $\pi_{35}=\frac{\sigma_{g o}}{\rho_{o}^{3 / 2} L^{2} T C_{p f}}$ & $\pi_{47}=\frac{k_{f}}{\rho_{o} L^{5 / 2} T^{1 / 2} C_{p f}^{5 / 2}}$ & $\pi_{59}=\frac{L_{v}}{L T C_{p f}}$ \\
\hline$\pi_{12}=\frac{k_{r}^{2}}{L^{2}}$ & $\pi_{24}=s_{g}$ & $\pi_{36}=\frac{\sigma_{o w}}{\rho_{o}^{3 / 2} L^{2} T C_{p f}}$ & $\pi_{48}=\frac{k_{g}}{\rho_{o} L^{5 / 2} T^{1 / 2} C_{p f}^{5 / 2}}$ & $\pi_{60}=\frac{\xi}{t}$ \\
\hline
\end{tabular}




\section{Mathematical formulation using inspectional analysis}

Let us consider the steam flooding process by considering modified Darcy's law for three-phase flows during the production. A model was derived using a rock and fluid memory concept for the development of the scaling criteria of the steam flooding process. The relationship between different process-controlling parameters was developed through the effective combination of those dimensionless groups. Finally, a model equation was developed for the displacement of oil by steam flood using modified Darcy's law by incorporating rock and fluid memory concepts (Hossain et al. 2007, 2008, 2009). The flow equation can be written as follows:

$u_{x}=-\eta\left[\frac{\partial^{\alpha}}{\partial t^{\alpha}}\left(\frac{\partial p}{\partial x}\right)\right]$

where $\frac{\partial^{\alpha}}{\partial t^{\alpha}}[p(x, t)]=\frac{1}{\Gamma(1-\alpha)} \int_{0}^{t}(t-\xi)^{-\alpha} \frac{\partial}{\partial \xi}[p(x, t)] \mathrm{d} \xi$, with $0 \leq \alpha<1$.

Equation (6) can be written as:

$u_{x}=-\frac{\eta}{\Gamma(1-\alpha)} \int_{0}^{t}(t-\xi)^{-\alpha} \frac{\partial^{2} p}{\partial \xi \partial x} \mathrm{~d} \xi$
Equation (7) can be written for oil, water, and gas phases in the direction of $x$ and $z$-axes.

$$
\begin{aligned}
& u_{x o}=-\frac{\eta_{o}}{\Gamma(1-\alpha)} \int_{0}^{t}(t-\xi)^{-\alpha} \frac{\partial^{2} p_{o}}{\partial \xi \partial x} \mathrm{~d} \xi \\
& u_{z o}=-\frac{\eta_{o}}{\Gamma(1-\alpha)} \int_{0}^{t}(t-\xi)^{-\alpha} \frac{\partial^{2} p_{o}}{\partial \xi \partial z} \mathrm{~d} \xi \\
& u_{x w}=-\frac{\eta_{w}}{\Gamma(1-\alpha)} \int_{0}^{t}(t-\xi)^{-\alpha} \frac{\partial^{2} p_{w}}{\partial \xi \partial x} \mathrm{~d} \xi \\
& u_{z w}=-\frac{\eta_{w}}{\Gamma(1-\alpha)} \int_{0}^{t}(t-\xi)^{-\alpha} \frac{\partial^{2} p_{w}}{\partial \xi \partial z} \mathrm{~d} \xi \\
& u_{x g}=-\frac{\eta_{g}}{\Gamma(1-\alpha)} \int_{0}^{t}(t-\xi)^{-\alpha} \frac{\partial^{2} p_{g}}{\partial \xi \partial x} \mathrm{~d} \xi
\end{aligned}
$$

$u_{z g}=-\frac{\eta_{g}}{\Gamma(1-\alpha)} \int_{0}^{t}(t-\xi)^{-\alpha} \frac{\partial^{2} p_{g}}{\partial \xi \partial z} \mathrm{~d} \xi$

Now the mass balance equations for different phases are written as

Mass balance of aqueous phase

$$
\begin{aligned}
& \frac{\partial}{\partial x} u_{x w} E_{w 1}+\frac{\partial}{\partial z} u_{z w} E_{w 1}+\frac{\partial}{\partial x} u_{x w} E_{w 3}+\frac{\partial}{\partial z} u_{z w} E_{w 3}+\frac{\partial}{\partial x} u_{x w} E_{w 4}+\frac{\partial}{\partial z} u_{z w} E_{w 4}+\phi E_{L w 1} \frac{\partial^{2}}{\partial x^{2}} E_{w 1} \\
& +\phi E_{T w 1} \frac{\partial^{2}}{\partial z^{2}} E_{w 1}+\phi D_{L w 3} \frac{\partial^{2}}{\partial x^{2}} E_{w 3}+\phi D_{T w 3} \frac{\partial^{2}}{\partial z^{2}} E_{w 3}+\phi D_{L w 4} \frac{\partial^{2}}{\partial x^{2}} E_{w 4}+\phi D_{T w 4} \frac{\partial^{2}}{\partial z^{2}} E_{w 4}=\phi \frac{\partial}{\partial t} s_{w} E_{w 1} \\
& \quad+\phi \frac{\partial}{\partial t} s_{w} E_{w 3}+\phi \frac{\partial}{\partial t} s_{w} E_{w 4}
\end{aligned}
$$

$$
\begin{aligned}
& \frac{\partial}{\partial x} u_{o x} E_{o 1}+\frac{\partial}{\partial z} u_{o z} E_{o 1}+\frac{\partial}{\partial x} u_{o x} E_{o 2}+\frac{\partial}{\partial z} u_{o z} E_{o 2}+\frac{\partial}{\partial x} u_{o x} E_{o 4}+\frac{\partial}{\partial z} u_{o z} E_{o 4}+\phi E_{L o 1} \frac{\partial^{2}}{\partial x^{2}} E_{o 1} \\
& \quad+\phi E_{T o 1} \frac{\partial^{2}}{\partial z^{2}} E_{o 1}+\phi E_{L o 2} \frac{\partial^{2}}{\partial x^{2}} E_{o 2}+\phi D_{T o 2} \frac{\partial^{2}}{\partial z^{2}} E_{o 2}+\phi D_{L o 4} \frac{\partial^{2}}{\partial x^{2}} E_{o 4}+\phi D_{T o 4} \frac{\partial^{2}}{\partial z^{2}} E_{o 4}=\phi \frac{\partial}{\partial t} s_{o} E_{o 1} \\
& \quad+\phi \frac{\partial}{\partial t} s_{o} E_{o 2}+\phi \frac{\partial}{\partial t} s_{o} E_{o 4}
\end{aligned}
$$


Table 2 Dimensionless groups from inspectional analysis

\begin{tabular}{|c|c|c|c|}
\hline $\begin{array}{l}\pi_{1}=\frac{t_{R} u_{x w R}}{\phi x_{R} s_{w R}} \\
\pi_{2}=\frac{t_{R} u_{z w R}}{\phi z_{R} s_{w R}} \\
\pi_{3}=\frac{D_{L w 1 R} t_{R}}{x_{R}^{2} s_{w R}}\end{array}$ & $\begin{aligned} \pi_{45} & =\frac{\eta_{g R} p_{\text {cowR }}}{\Gamma(1-\alpha) t_{R}^{\alpha-1} \phi_{R} s_{w R} x_{R}^{2}} \\
\pi_{46} & =\frac{f_{x o R} \eta_{o R} p_{\text {cowR }}}{\Gamma(1-\alpha) t_{R}^{\alpha-1} \phi_{R} s_{w R} x_{R}^{2}} \\
\pi_{47} & =\frac{f_{x o R} \eta_{g R} p_{\text {cow } R}}{\Gamma(1-\alpha) t_{R}^{\alpha-1} \phi_{R} s_{w R} x_{R}^{2}}\end{aligned}$ & $\begin{aligned} \pi_{89} & =\frac{\eta_{w R} p_{w R}}{\Gamma(1-\alpha) t_{R}-1} \phi_{R} s_{w R} z_{R}^{2} \\
\pi_{90} & =\frac{\eta_{g R} p_{w R}}{\Gamma(1-\alpha) t_{R}^{\alpha-1} \phi_{R} s_{w R} z_{R}^{2}} \\
\pi_{91} & =\frac{f_{x o R} \eta_{o R} p_{w R}}{\Gamma(1-\alpha) t_{R}^{-1} \phi_{R} s_{w R} z_{R}^{2}}\end{aligned}$ & $\begin{array}{l}\pi_{133}=\frac{s_{g i}}{s_{g R}} \\
\pi_{134}=\frac{s_{o i}}{s_{o R}} \\
\pi_{135}=\frac{E_{2 i}}{E_{o 2 R}}\end{array}$ \\
\hline$\pi_{4}=\frac{D_{T_{w 1 R} t_{R}}}{z_{R}^{2} s_{w R}}$ & $\pi_{48}=\frac{f_{x w R} \eta_{o R} p_{c o w R}}{\Gamma(1-\alpha) t_{R}^{\alpha-1} \phi_{R} s_{w R} x_{R}^{2}}$ & $\pi_{92}=\frac{f_{x o R} \eta_{w R} p_{w R}}{\Gamma(1-\alpha) t_{R}^{\alpha-1} \phi_{R} s_{w R}^{2} z_{R}^{2}}$ & $\pi_{136}=\frac{E_{3 i}}{E_{w 3 R}}$ \\
\hline$\pi_{5}=\frac{D_{L w 3 R} t_{R}}{x_{R}^{s} s_{w R}}$ & $\pi_{49}=\frac{f_{x w R} \eta_{o R} p_{c o w R}}{\Gamma(1-\alpha) t_{R}^{\alpha-1} \phi_{R} s_{w R} x_{R}^{2}}$ & $\pi_{93}=\frac{f_{x o R} \eta_{g R} p_{w R}}{\Gamma(1-\alpha) t_{R}^{\alpha-1} \phi_{R} s_{w R} z_{R}^{2}}$ & $\pi_{137}=\frac{E_{1 j}}{E_{g 1 R}}$ \\
\hline$\pi_{6}=\frac{D_{T_{w 3 R} t_{R}}}{z_{R}^{2} s_{w R}}$ & $\pi_{50}=\frac{\eta_{g R} p_{c g o R}}{\Gamma(1-\alpha) t_{R}^{\alpha-1} \phi_{R} s_{w R} x_{R}^{2}}$ & $\pi_{94}=\frac{f_{x w R} \eta_{o R} p_{w R}}{\Gamma(1-\alpha) t_{R}^{\alpha-1} \phi_{R} s_{w R} z_{R}^{2}}$ & $\pi_{138}=\frac{E_{4 j}}{E_{w 4 R}}$ \\
\hline$\pi_{7}=\frac{D_{L w 4 R} t_{R}}{x_{R} s_{w R}}$ & $\pi_{51}=\frac{f_{x o R} \eta_{g R} p_{c g o R}}{\Gamma(1-\alpha) t_{R}^{\alpha-1} \phi_{R} s_{w R} x_{R}^{2}}$ & $\pi_{95}=\frac{f_{x w R} \eta_{\eta_{w R}} p_{w R}}{\Gamma(1-\alpha) t_{R}^{-1-1} \phi_{R} s_{w R} z_{R}^{2}}$ & $\pi_{139}=\frac{\Delta p_{g R}}{p_{g R}}$ \\
\hline$\pi_{8}=\frac{D_{T_{w 4 R} t_{R}}}{z_{R}^{2} s_{w R}}$ & $\pi_{52}=\frac{f_{x w R} \eta_{g R} p_{c g o R}}{\Gamma(1-\alpha) t_{R}^{\alpha-1} \phi_{R} s_{w R} x_{R}^{2}}$ & $\pi_{96}=\frac{f_{x w R} \eta_{g R} p_{w R}}{\Gamma(1-\alpha) t_{R}^{\alpha-1} \phi_{R} s_{w R} z_{R}^{2}}$ & $\pi_{140}=\frac{\rho_{g R} g_{R} H_{R}}{p_{g R}}$ \\
\hline$\pi_{9}=\frac{t_{R} u_{x o R}}{\phi x_{R} s_{o R}}$ & $\pi_{53}=\frac{p_{w R}}{p_{c o w R}}$ & $\pi_{97}=\frac{\eta_{o R} p_{c o w R}}{\Gamma(1-\alpha) t_{R}^{\alpha-1} \phi_{R} s_{w R} z_{R}^{2}}$ & $\pi_{141}=\frac{\rho_{g R} g_{R} z_{R}}{p_{g R}}$ \\
\hline$\pi_{10}=\frac{t_{R} u_{z o R}}{\phi z_{R} s_{o R}}$ & $\pi_{54}=\frac{x_{R}^{2}}{z_{R}^{2}}$ & $\pi_{98}=\frac{\eta_{g R} p_{\text {cow } R}}{\Gamma(1-\alpha) t_{R}^{\alpha-1} \phi_{R} s_{w R}^{2} z_{R}^{2}}$ & $\pi_{142}=\frac{\Delta p_{o R}}{p_{o R}}$ \\
\hline$\pi_{11}=\frac{D_{L o 1 R} t_{R}}{x_{R}^{2} s_{o R}}$ & $\pi_{55}=\frac{p_{w R} x_{R}^{2}}{p_{\text {cow }} z_{R}^{2}}$ & $\pi_{99}=\frac{f_{x o R} \eta_{o R} p_{c o w R}}{\Gamma(1-\alpha) t_{R}^{\alpha-1} \phi_{R} s_{w R} z_{R}^{2}}$ & $\pi_{143}=\frac{\rho_{o R} g_{R} H_{R}}{p_{o R}}$ \\
\hline$\pi_{12}=\frac{D_{T O 1 I_{R}} t_{R}}{z_{R}^{2} s_{o R}}$ & $\pi_{56}=\frac{p_{w R}}{p_{c g o R}}$ & $\pi_{100}=\frac{f_{x o R} \eta_{g R} p_{\text {cowR }}}{\Gamma(1-\alpha) t_{R}^{\alpha-1} \phi_{R} s_{w R} z_{R}^{2}}$ & $\pi_{144}=\frac{\rho_{o R} g_{R} z_{R}}{p_{o R}}$ \\
\hline$\pi_{13}=\frac{D_{L o 2 R} t_{R}}{x_{R}^{2} s_{o R}}$ & $\pi_{57}=\frac{p_{w R} x_{R}^{2}}{p_{c g o R} z_{R}^{2}}$ & $\pi_{101}=\frac{f_{x w R} \eta_{o R} p_{c o w R}}{\Gamma(1-\alpha) t_{R}^{\alpha-1} \phi_{R} s_{w R} z_{R}^{2}}$ & $\pi_{145}=\frac{\Delta p_{w R}}{p_{w R}}$ \\
\hline$\pi_{14}=\frac{D_{T o 2 R} t_{R}}{z_{R}^{2} s_{o R}}$ & $\pi_{58}=\frac{p_{\text {cow } R}}{p_{\text {cgoR }}}$ & $\pi_{102}=\frac{f_{x w R} \eta_{o R} p_{c o w R}}{\Gamma(1-\alpha) t_{R}^{\alpha-1} \phi_{R} s_{w R} z_{R}^{2}}$ & $\pi_{146}=\frac{\rho_{w R} g_{R} H_{R}}{p_{w R}}$ \\
\hline$\pi_{15}=\frac{D_{L o t R} t_{R}}{x_{R}^{2} s_{o R}}$ & $\pi_{59}=\frac{p_{\text {cowR }} x_{R}^{2}}{p_{\text {cgoR }} z_{R}^{2}}$ & $\pi_{103}=\frac{\eta_{g R} p_{c g o R}}{\Gamma(1-\alpha) t_{R}^{\alpha-1} \phi_{R} S_{w R} z_{R}^{2}}$ & $\pi_{147}=\frac{\rho_{w R} g_{R} z_{R}}{p_{w R}}$ \\
\hline$\pi_{16}=\frac{D_{T o 4 t_{R}} t_{R}}{z_{R}^{2} s_{o R}}$ & $\pi_{60}=\frac{f_{x o R} p_{w R}}{p_{c o w R}}$ & $\pi_{104}=\frac{f_{x o R} \eta_{g R} p_{c g o R}}{\Gamma(1-\alpha) t_{R}^{\alpha-1} \phi_{R} s_{w R} z_{R}^{2}}$ & $\pi_{148}=\frac{H U_{t}}{z_{R} u_{g \times R} z_{R}}$ \\
\hline$\pi_{17}=\frac{u_{x g R} t_{R}}{\phi x_{R} s_{g R}}$ & $\pi_{61}=\frac{f_{x o R} x_{R}^{2}}{z_{R}^{2}}$ & $\pi_{105}=\frac{f_{x w R} \eta_{g R} p_{c g o R}}{\Gamma(1-\alpha) t_{R}^{\alpha-1} \phi_{R} s_{w R} z_{R}^{2}}$ & $\pi_{149}=\frac{z_{R}^{2} D_{L o 1 R}}{x_{R}^{2} D_{T o 1 R}}$ \\
\hline$\pi_{18}=\frac{u_{z g R} t_{R}}{\phi z_{R} s_{g R}}$ & $\pi_{62}=\frac{f_{x o R} p_{w R} x_{R}^{2}}{p_{c o w R} z_{R}^{2}}$ & $\pi_{106}=\frac{t_{R} D_{L w 1 R}}{s_{w R} x_{R}^{2}}$ & $\pi_{150}=\frac{z_{R}^{2} D_{L o 2 R}}{x_{R}^{2} D_{T o 2 R}}$ \\
\hline$\pi_{19}=\frac{u_{x o R}}{U_{x R}}$ & $\pi_{63}=\frac{f_{x o R} p_{w R}}{p_{c g o R}}$ & $\pi_{107}=\frac{t_{R} D_{L w 3 R}}{s_{w R} x_{R}^{2}}$ & $\pi_{151}=\frac{z_{R}^{2} D_{L o 4 R}}{x_{R}^{2} D_{T o 4 R}}$ \\
\hline$\pi_{20}=\frac{u_{x w R}}{U_{x R}}$ & $\pi_{64}=\frac{f_{x o R} p_{w R} x_{R}^{2}}{p_{c g o R} z_{R}^{2}}$ & $\pi_{108}=\frac{t_{R} D_{L w 4 R}}{s_{w R} x_{R}^{2}}$ & $\pi_{152}=\frac{z_{R}^{2} D_{L w 1 R}}{x_{R}^{2} D_{T w 1 R}}$ \\
\hline$\pi_{21}=\frac{u_{x g R}}{U_{x R}}$ & $\pi_{65}=\frac{f_{x o R} p_{c o w R}}{p_{c g o R}}$ & $\pi_{109}=\frac{t_{R} D_{T w 1 R}}{s_{w R} z_{R}^{2}}$ & $\pi_{153}=\frac{z_{R}^{2} D_{L w 2 R}}{x_{R}^{2} D_{T w 2 R}}$ \\
\hline$\pi_{22}=\frac{u_{z o R}}{U_{z R}}$ & $\pi_{66}=\frac{f_{\text {xoR }} p_{\text {cowR }} x_{R}^{2}}{p_{\text {cgoR }} z_{R}^{2}}$ & $\pi_{110}=\frac{t_{R} D_{T w 3 R}}{s_{W R} z_{R}^{2}}$ & $\pi_{154}=\frac{z_{R}^{2} D_{L w 4 R}}{x_{R}^{2} D_{T w}}$ \\
\hline$\pi_{23}=\frac{u_{z w R}}{U_{z R}}$ & $\pi_{67}=\frac{f_{x w R} p_{w R}}{p_{c o w R}}$ & $\pi_{111}=\frac{t_{R} D_{T_{w} 4 R}}{s_{w R} z_{R}^{2}}$ & $\pi_{155}=\frac{H}{z_{R}}$ \\
\hline$\pi_{24}=\frac{u_{z g R}}{U_{z R}}$ & $\pi_{68}=\frac{f_{x w R} x_{R}^{2}}{z_{R}^{2}}$ & $\pi_{112}=\frac{k_{x w R} \lambda_{x w R} p_{w R}}{x_{R} u_{x w R}}$ & $\pi_{156}=\frac{L}{x_{R}}$ \\
\hline$\pi_{25}=\frac{u_{x o R}}{f_{x o R} U_{x R}}$ & $\pi_{69}=\frac{f_{x w R} p_{w R} x_{R}^{2}}{f_{x w R} p_{c o w R} z_{R}^{2}}$ & $\pi_{113}=\frac{k_{x w R} \lambda_{x w R} \rho_{w R} g_{R} z_{R} \sin \theta_{R}}{x_{R} u_{x w R}}$ & $\pi_{157}=\frac{H U_{t}}{u_{g \times R} z_{R}}$ \\
\hline$\pi_{26}=\frac{u_{z o R}}{f_{z o R} U_{z R}}$ & $\pi_{70}=\frac{f_{x w R} p_{w R}}{p_{c g o R}}$ & $\pi_{114}=\frac{k_{x o R} \lambda_{x o R} p_{o R}}{x_{R} u_{x o R}}$ & $\pi_{158}=\frac{K_{h r} T A t}{L \Delta T M_{1} V}$ \\
\hline$\pi_{27}=\frac{u_{x w R}}{f_{x w R} U_{x R}}$ & $\pi_{71}=\frac{f_{x w R} p_{w R} x_{R}^{2}}{p_{c g o R} z_{R}^{2}}$ & $\pi_{115}=\frac{k_{x o R} \lambda_{x o R} \rho_{o R} g_{R} z_{R} \sin \theta_{R}}{x_{R} u_{x o R}}$ & $\pi_{159}=\frac{K_{h f} T A t}{L \Delta T M_{1} V}$ \\
\hline$\pi_{28}=\frac{u_{z w R}}{f_{z w R} U_{z R}}$ & $\pi_{72}=\frac{f_{x w R} p_{c o w R}}{p_{c g o R}}$ & $\pi_{116}=\frac{k_{x g R} \lambda_{x g R} p_{g R}}{x_{R} u_{x g R}}$ & $\pi_{160}=\frac{t C_{p o} \rho_{o} u_{o x} A}{M_{1} V}$ \\
\hline$\pi_{29}=\frac{u_{x g R}}{f_{x g R} U_{x R}}$ & $\pi_{73}=\frac{f_{\text {swR }} p_{\text {cow }} x_{R}^{2}}{p_{\text {cgoR }}^{2} z_{R}^{2}}$ & $\pi_{117}=\frac{k_{x g R} \lambda_{x g R} \rho_{g R} g_{R} z_{R} \sin \theta_{R}}{x_{R} u_{x g R}}$ & $\pi_{161}=\frac{t C_{p w} \rho_{w} u_{w x} A}{M_{1} V}$ \\
\hline$\pi_{30}=\frac{f_{x o R}}{f_{x g R}}$ & $\pi_{74}=\frac{f_{z o R} p_{w R} x_{R}^{2}}{p_{\text {cowR }} z_{R}^{2}}$ & $\pi_{118}=\frac{k_{z w R} \lambda_{z w R} p_{w R}}{z_{R} u_{z w R}}$ & $\pi_{162}=\frac{t C_{p o} \rho_{o} \phi S_{o} v_{x} A}{M_{1} V}$ \\
\hline$\pi_{31}=\frac{f_{x w R}}{f_{x g R}}$ & $\pi_{75}=\frac{f_{z o R} p_{w R}}{p_{c g o R}}$ & $\pi_{119}=\frac{k_{z w R} \lambda_{z w R} \rho_{w R} g_{R} z_{R} \sin \theta_{R}}{z_{R} u_{x w R}}$ & $\pi_{163}=\frac{t C_{p w} \rho_{w} \phi S_{w} v_{x} A}{M_{1} V}$ \\
\hline
\end{tabular}


Table 2 (continued)

\begin{tabular}{|c|c|c|c|}
\hline$\pi_{32}=\frac{u_{z g R}}{f_{z B R} U_{z R}}$ & $\pi_{76}=\frac{f_{z o R} p_{w R} x_{R}^{2}}{p_{c o w R} z_{R}^{2}}$ & $\pi_{120}=\frac{k_{z o R} \lambda_{z o R} p_{o R}}{z_{R} u_{z o R}}$ & $\pi_{164}=\frac{(1-\phi) C_{p r} \rho_{r} v_{x} A}{\Delta T M_{1} V}$ \\
\hline$\pi_{33}=\frac{f_{z o R}}{f_{z g R}}$ & $\pi_{77}=\frac{f_{z o R} p_{w R}}{p_{c g o R}}$ & $\pi_{121}=\frac{k_{z o R} \lambda_{z o R} \rho_{o R} g_{R} z_{R} \sin \theta_{R}}{z_{R} u_{z o R}}$ & $\pi_{165}=\frac{m_{s} f_{s} L_{v} t}{\Delta T M_{1} V}$ \\
\hline$\pi_{34}=\frac{f_{z w R}}{f_{z g R}}$ & $\pi_{78}=\frac{f_{z o R} p_{w R} x_{R}^{2}}{p_{c g o R} z_{R}^{2}}$ & $\pi_{122}=\frac{k_{z g R} \lambda_{z g R} p_{w R}}{z_{R} u_{z g R}}$ & $\pi_{166}=\frac{m_{s} C_{p w} t}{M_{1} V}$ \\
\hline$\pi_{35}=\frac{\eta_{o R} p_{w R}}{\Gamma(1-\alpha) t_{R}^{\alpha-1} \phi_{R} s_{w R} x_{R}^{2}}$ & $\pi_{79}=\frac{f_{z o R} p_{c o w R}}{p_{c g o R}}$ & $\pi_{123}=\frac{k_{z g R} \lambda_{z g R} \rho_{g R} g_{R} z_{R} \sin \theta_{R}}{z_{R} u_{z g R}}$ & $\pi_{167}=\frac{(1-\phi) \rho_{r} C_{p r}}{M_{1}}$ \\
\hline$\pi_{36}=\frac{\eta_{w R} p_{w R}}{\Gamma(1-\alpha) t_{R}^{\alpha-1} \phi_{R} s_{w R} x_{R}^{2}}$ & $\pi_{80}=\frac{f_{x o R} p_{\text {cowR }} x_{R}^{2}}{p_{c g o R} z_{R}^{2}}$ & $\pi_{124}=\frac{\sigma_{\text {owR }} J_{R}\left(s_{w}\right) \cos \theta_{R} \sqrt{\frac{\phi_{R}}{k_{R}}}}{p_{\text {cowR }}}$ & $\pi_{168}=\frac{\phi C_{p w} \rho_{w} S_{w}}{M_{1}}$ \\
\hline$\pi_{37}=\frac{\eta_{g R} p_{w R}}{\Gamma(1-\alpha) t_{R}^{\alpha-1} \phi_{R} s_{w R} x_{R}^{2}}$ & $\pi_{81}=\frac{f_{x w R} p_{w R}}{p_{c o w R}}$ & $\pi_{125}=\frac{\sigma_{g o R} J_{R}\left(s_{g}\right) \cos \theta_{R} \sqrt{\frac{\phi_{R}}{k_{R}}}}{p_{c g o R}}$ & $\pi_{169}=\frac{\phi c_{p o} \rho_{o} S_{0}}{M_{1}}$ \\
\hline$\pi_{38}=\frac{f_{x o R} \eta_{o R} p_{w R}}{\Gamma(1-\alpha))_{R}^{t-1} \phi_{R} s_{w R} x_{R}^{2}}$ & $\pi_{82}=\frac{f_{x w R} x_{R}^{2}}{z_{R}^{2}}$ & $\pi_{126}=\frac{s_{o R}}{s_{w R}}$ & $\pi_{170}=\frac{\phi C_{p g} \rho_{g} S_{g}}{M_{1}}$ \\
\hline 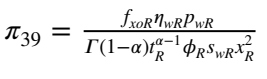 & $\pi_{83}=\frac{f_{x w R} p_{w R} x_{R}^{2}}{f_{x w R} p_{c o w R} z_{R}^{2}}$ & $\pi_{127}=\frac{s_{g R}}{s_{w R}}$ & $\pi_{171}=\frac{\phi L_{v} \rho_{g} S_{g}}{M_{1} \Delta T}$ \\
\hline$\pi_{40}=\frac{f_{x o R} \eta_{g R} p_{w R}}{\Gamma(1-\alpha))_{R}^{t-1} \phi_{R} s_{w R} x_{R}^{2}}$ & $\pi_{84}=\frac{f_{x w R} p_{w R}}{p_{c g o R}}$ & $\pi_{128}=\frac{s_{o}-s_{o r}}{1-s_{w i}-s_{o r}}$ & $\pi_{172}=\frac{K_{h w} S_{w}}{K_{h f}}$ \\
\hline$\pi_{41}=\frac{f_{x w R} \eta_{o R} p_{w R}}{\Gamma(1-\alpha))_{R}^{t-1} \phi_{R} s_{w R} x_{R}^{2}}$ & $\pi_{85}=\frac{f_{x w R} p_{w R} x_{R}^{2}}{p_{c g o R} Z_{R}^{2}}$ & $\pi_{129}=\frac{s_{w}-s_{w i}}{1-s_{w i}-s_{o r}}$ & $\pi_{173}=\frac{K_{h o} S_{o}}{K_{h f}}$ \\
\hline$\pi_{42}=\frac{f_{x w R} \eta_{w R} p_{w R}}{\Gamma(1-\alpha) t_{R}^{t-1} \phi_{R} s_{w R} x_{R}^{2}}$ & $\pi_{86}=\frac{f_{x w R} p_{c o w R}}{p_{c g o R}}$ & $\pi_{130}=\frac{s_{g}-s_{g c}}{1-s_{w i}-s_{o r}}$ & $\pi_{174}=\frac{K_{h g} S_{g}}{K_{h f}}$ \\
\hline$\pi_{43}=\frac{f_{x w R} \eta_{g R} p_{w R}}{\Gamma(1-\alpha) t_{R}^{t-1} \phi_{R} s_{w R} x_{R}^{2}}$ & $\pi_{87}=\frac{f_{x w R} p_{c o w R} x_{R}^{2}}{p_{c g o R} z_{R}^{2}}$ & $\pi_{131}=\frac{U_{t} t}{L \phi\left(1-s_{w i}-s_{o r}\right)}$ & $\pi_{175}=\frac{\phi K_{h f}}{K_{h e}}$ \\
\hline$\pi_{44}=\frac{\eta_{o R} p_{c o w R}}{\Gamma(1-\alpha) t_{R}^{t-1} \phi_{R} s_{w R} x_{R}^{2}}$ & $\pi_{88}=\frac{\eta_{o R} p_{w R}}{\Gamma(1-\alpha) t_{R}^{\alpha-1} \phi_{R} s_{w R} z_{R}^{2}}$ & $\pi_{132}=\frac{s_{w i}}{s_{w D}}$ & $\pi_{176}=\frac{(1-\phi) K_{h r}}{K_{h e}}$ \\
\hline
\end{tabular}

Mass balance of gaseous phase

$\frac{\partial}{\partial x} u_{x g} E_{g 1}+\frac{\partial}{\partial z} u_{z g} E_{g 1}=\phi \frac{\partial}{\partial t} s_{g} E_{g 1}$

Thermal energy balance equation can be written in integral form over the steam zone modified from Yortsos (1979)

\section{Dimensionless groups}

The dimensionless groups from the inspectional analysis are given in Table 2. Different assumptions, initial and boundary conditions, are used along with constitutive relationships and constraints to derive those groups. Capillary number,

$$
\begin{aligned}
& \Delta T \frac{\mathrm{d}}{\mathrm{d} t} \int_{v(t)} M_{1} d v+\int_{A_{c a(t)}}\left(-K_{h c} \frac{\partial T}{\partial x}\right) \mathrm{d} A+\int_{A_{s f(t)}}\left(-K_{h} \frac{\partial T}{\partial x}\right) \mathrm{d} A+\Delta T\left[\sum_{i=w, o} C_{i} \int_{A_{s f(t)}} \rho_{i}\left(u_{i x}-\phi s_{i} v_{x}\right) \mathrm{d} A\right. \\
& \left.-(1-\phi) C_{r} \int_{A_{s f(t)}} \rho_{r} v_{x} \mathrm{~d} A\right]=m_{s}\left[f_{s} L_{v}+C_{w} \Delta T\right]
\end{aligned}
$$

Here volumetric integral covers the steam zone volume $v(t)$, and the areal integrals are evaluated over steam front area $A_{f(t)}$ to the steam zone area $A_{c(t)}$ contacting adjacent formation. $\frac{\partial T}{\partial x}$ represents the temperature gradient normal to the steam front, $v_{x}$ is the steam front velocity, and $M_{1}$ is the volumetric heat capacity of the steam zone.

$M_{1}=(1-\phi) \rho_{r} c_{r}+\phi\left(c_{w} \rho_{w} s_{w}+c_{o} \rho_{o} s_{0}+c_{g} \rho_{g} s_{g}\right)+\frac{\phi L_{v} \rho_{g} s_{g}}{\Delta T}$ gravity number, geometric aspect ratio, longitudinal and transverse Peclet number, dimensionless time, conductivity ratio, dispersion factor, mobility ratio, and other groups are developed using inspectional analysis. These are the primary dimensionless groups derived from the steam flooding process. After the primary, secondary, and tertiary elimination of groups, the desired dimensionless groups will be found, which will ultimately affect the steam flooding process. 


\section{High Pressure Scaling Reservoir Fluids}

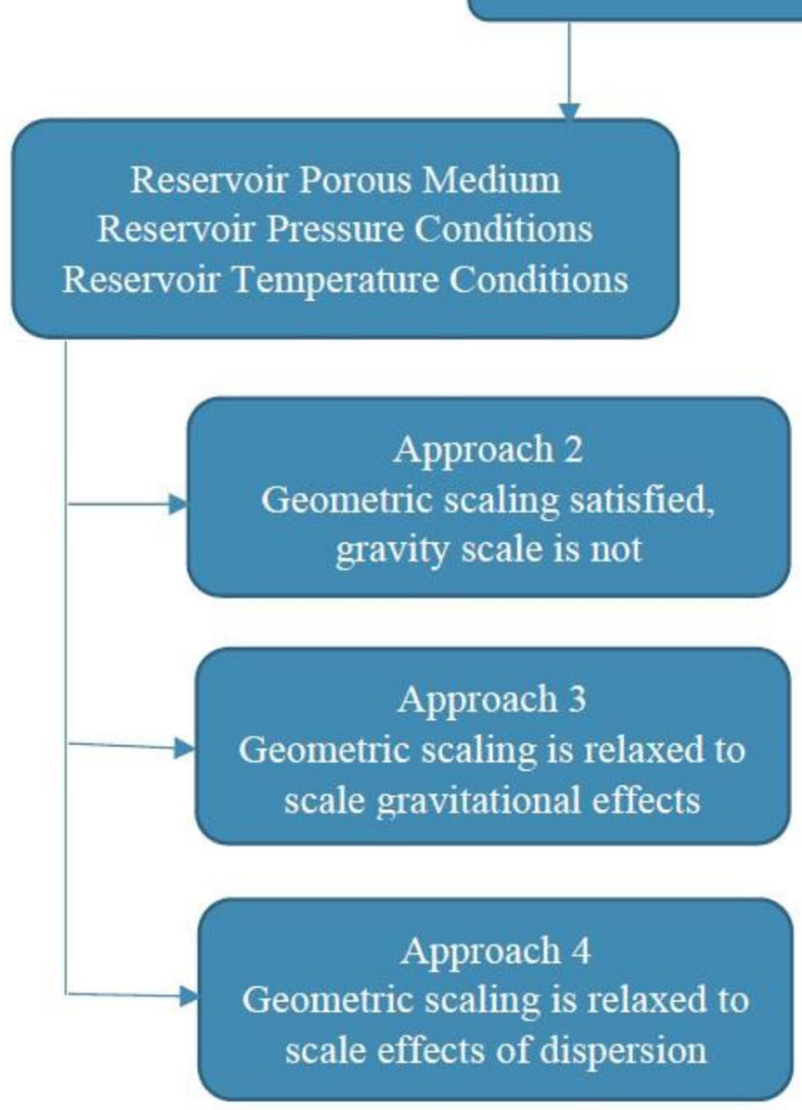

Fig. 2 Scaling approaches

\section{Approaches to satisfy scaling groups}

The dimensionless groups derived in the previous section should be satisfied with the scaling process, which is governed by the governing equations, initial and boundary conditions, constitutive relationships, and constraints. The similarity groups should be analogous in model and prototype. It is very difficult to satisfy a complete set of scaling criteria, so several groups should be relaxed to fulfil all the requirements of scaling criteria. Figure 1 describes the different approaches to satisfy a specific process. These approaches are applicable to high-pressure reservoir fluids, where different reservoir pressure-temperature conditions are used. Approaches 2, 3, and 4 are used for a porous medium with reservoir pressure and temperature conditions, while approaches 1 and 5 are applicable for pressure and temperature conditions (Fig. 2).
Different Porous Media

Different Pressure Drops

Same Initial Temperature

\section{Approach 1}

Geometric scaling is satisfied,

pressure-temperature relationship

is not

\section{Approach 5}

Geometric scaling is relaxed to

scale effects of dispersion

\section{Approach 1: same fluid, different porous media, different pressure drop, and geometric similarity}

Geometric similarity groups can be satisfied by considering pressure drop, gravitational, and viscous forces, which are different for model and prototype. This condition requires different porous media. Pujol and Boberg (1972) proposed this approach, which allows scaling requirements should be satisfied if it violates some constitutive relationships, constraints, and boundary conditions. Saturation pressure and saturation temperature relationship for the steam flooding process cannot be properly scaled by this method, which will ultimately mislead the heat losses from the steam zone. Different steam properties which largely depend on pressure will not be scaled properly. Since a different porous medium is considered, the fluid saturations and relative permeability are not scaled accurately. In addition, capillary 
forces and dispersion effects are not properly scaled using this approach.

The implementation of these scaling criteria for a model can reduce the length by a scaling factor of $a$.

1. The value of $\phi, s_{w}, s_{o}, s_{g}, E_{w}, E_{g}, T, \Delta T$ remains the same;

2. The values of $H, \Delta p$ should be reduced by a factor of $a$;

3. The value of $k$ should be increased by a factor of $a$;

4. The value of $t$ should be reduced by a factor of $a^{2}$

If the gravitational force is important, this technique is suitable only for the steam flooding process. This approach is unable to scale additive accurately. Relaxed and satisfied scaling groups are given in Table 3 .

\section{Approach 2: same fluids, same pressure drop, same porous medium, geometric similarity}

The difficulties raised in approach 1 should be overcome by considering the maximum pressure and temperature difference along with the initial pressure, and the temperature remains the same for model and prototype. This assumption has allowed the properties to depend on pressure and temperature, which are properly scaled. As the same porous medium is used here, the fluid saturations and relative permeabilities are properly scaled. In addition, viscous forces, diffusion effects, and heat transfers are properly scaled due to these changes. The limitation of this approach is that it cannot accurately scale gravitational forces. Another limitation is that it cannot scale dispersion effects if the flow rate is very high.

The implementation of these scaling criteria for a model can reduce the length by a scaling factor of ' $a$ '.

1. The value of $\phi, s_{w}, s_{o}, s_{g}, \Delta p, T, \Delta T$ remain same;

2. The values of $H, U_{t}$ should be reduced by a factor of $a$;

3. The value of $t$ should be reduced by a factor of $a^{\left(\frac{2}{\alpha-1}\right)}$

This approach is restricted to processes where the gravitational force is not much important such as thin formations with high flow rates. Diffusion effects and PVT properties scale well for steam flooding used with additives. Relaxed and satisfied scaling groups are given in Table 3 .

\section{Approach 3: same fluids, same pressure drop, same porous media, relaxed geometric similarity}

The advantage of using the same porous medium, same fluid, and similar pressure and temperature conditions helps to scale gravitational forces properly but allows the geometric similarity to be relaxed. If the pressure gradient is low due to capillary and viscous forces, capillary and viscous forces can be scaled for the horizontal well. The vertical direction heat conduction, dispersion effects, and capillary number are not properly scaled.

The implementation of these scaling criteria for a model can reduce the length by a scaling factor of ' $a$ '. The value of $\phi, s_{w}, s_{o}, s_{g}, \Delta p, p_{w R}, T, \Delta T$ remain the same.

1. The values of $H$ should be reduced by a factor of ' $a$ ';

2. The reservoir should be horizontal;

3. The value of $t$ should be reduced by a factor of $a^{\left(\frac{2}{\alpha-1}\right)}$

This approach is restricted to steam and steam additive processes where a significant reduction of reservoir thickness is considered. Relaxed and satisfied scaling groups are given in Table 3.

\section{Approach 4: same fluids, same porous media, same pressure drop, relaxed geometric similarity}

The previous approaches had not attempted to consider the dispersion effect for the case of high flow rates. It is difficult to scale dispersion effects. The objective of this approach is to scale transverse dispersion effects. Gravitational and capillary effects are not properly scaled, but viscous and dispersion effects are scaled properly. The merit of this approach is to satisfy all other dimensionless numbers and boundary conditions except capillary and gravity forces. This rigorous method is not very suitable when scaling the steam flooding process is involved.

The implementation of these scaling criteria for a model can reduce the length by a scaling factor of ' $a$ '.

1. The value of $\phi, s_{w}, s_{o}, s_{g}, \Delta p p_{w R}, T, \Delta T, k$ remain same;

2. The values of $H$ should be reduced by a factor of $a$;

3. The values of $U_{t}$ should be reduced by a factor of $a^{\left(\frac{1}{2}\right)}$;

4. The value of $t$ should be reduced by a factor of $a^{2}$

This approach is restricted to a process where dispersion effects are considered by relaxing gravitational forces. Hot water flooding with a liquid additive is a good option for this approach. In addition, this approach is restricted to thin formations because the only small reduction in thickness is considered. Relaxed and satisfied scaling groups are given in Table 3.

\section{Approach 5: same fluid, different pressure drops, different porous media, relaxed geometric similarity}

The main shortcoming of approach 4 is the relaxation of gravitational forces, but this approach tries to satisfy viscous and gravitational forces while still scaling transverse dispersion effects. As a different porous medium and different pressure drops are used, the limitation of approach 1 comes into place. Here, time is scaled by a four-fifth power 
of the scaling factor rather than squares indicating the longer period of experimental time. Capillary forces and heat conduction are not properly scaled. In addition, saturation pressure and boundary temperature for the steam flood is poorly scaled. Relaxed and satisfied scaling groups are given in Table 3.

The implementation of these scaling criteria for a model can reduce the length by a scaling factor of ' $a$ '.

1. The value of $\phi, s_{w}, s_{o}, s_{g}, T, \Delta T$ remain the same;

2. The values of $H$ should be reduced by a factor of $a$;

3. The values of $k$ should be increased by a factor of $a$;

4. The value of $t$ should be reduced by a factor of $a^{2}$.

\section{Comparison of different scaling approaches}

Table 2 lists dimensionless numbers and how their effects can change the model for the steam flooding process. Approach 1 can be used by numerous previous researchers. This approach can accurately have scaled the ratio of viscous to gravitation forces, but it had faced difficulty in scaling saturation temperature, saturation pressure, steam injection rate, steam density, the energy stored in the steam phase, and latent heat of vaporization. As different porous media are used, the relative permeabilities and irreducible saturation can also alter depending on the type of porous media.

Approach 2 would be the most suitable approach for the steam flooding process where gravitational force does not play a vital role. When the process is dominated by viscous force, this approach comes into play a vital role. The effects of gravitational force have been reduced in the model by employing this approach. When this approach creates a significant error under certain conditions which are not studied well, it is restricted to certain conditions for steam flooding process. In a study of the immiscible isothermal displacement of heavy oil by $\mathrm{CO}_{2}$ flooding, Rojas (1985) found that the recovery of oil is independent of the model prototype ratio when gravitational force-to-viscous force ratio is less than 5 . There should be upper limit and lower limit for this method. The upper limit may represent a point where dispersion effects can be scaled more effectively. On the other hand, the lower limit of gravitational forces can be scaled more rigorously.

Approach 3 can overcome the limitations of method 1. As the same fluid and same porous medium are used, it can ensure the irreducible saturation, and relative permeabilities can be scaled properly. It can also properly scale saturation temperature, saturation pressure, steam injection rate, steam density, the energy stored in the steam phase, and latent heat of vaporization. This approach cannot scale capillary forces along with the vertical conduction of energy. Baker $(1969,1973)$ investigated that heat losses are a function of time only and found that it did not depend on the injection rate. The effect of transverse dispersion effects will be enhanced in the model.
Approach 4 can easily scale the transverse dispersion effects, but gravitational and capillary forces are poorly scaled. It cannot scale the vertical conduction of energy properly. The scaling requirements of irreducible saturation, relative permeabilities, steam density, the heat stored in steam, saturation temperature-saturation pressure relationship, and injection temperature are satisfied.

Approach 5 satisfied the requirements for gravitational forces and balanced it with dispersive and viscous forces. However, it has several drawbacks, like other approaches. It required a significant reduction in pressure drop as well as a reduction in time scale factor to satisfy the scale conduction. Therefore, approach 5 poorly scales conduction.

The various aspects of recovery are largely depended on the selection of appropriate approaches. The selection of an appropriate approach is particularly dependent on the properties which are involved within this approach. In approaches 2,3 , and 4 , the same fluid, same pressure drops, and the same porous medium are used, but the temperature change has a significant effect in simulating these properties even though they have not been properly scaled. There are some important phenomena, such as gas solubility, emulsification, distillation, which are not considered here during the scaling process. The significance of a phenomenon is used as a selection criterion that is not scaled by the selected approach. If capillary force is a prime factor for a process, it is unlikely that approaches 3 and 4 would be satisfied. Similarly, for the case of gravitational forces, approaches 2 and 4 would not be satisfied. Another issue is the selection of relative significance of a phenomenon. If a phenomenon is considered insignificant for a process, it should remain insignificant in the model also. For selecting a scaling process, the effects of transverse dispersion are considered to be minor in prototype for the case of approach 3. If it remains insignificant in the model, then it would be considered as a suitable approach for this process.

\section{Conclusions}

A complete set of dimensionless groups is derived for the steam flooding process using dimensional and inspectional analyses. Modified Darcy equation is used for incorporating rock and fluid memory, constitutive relationships, constraints, initial and boundary conditions to develop the dimensionless groups. As all the requirements are not satisfied with a process, some of the dimensionless groups should be relaxed to satisfy a specific mechanism. Five sets of scaling approaches are selected, and each set consists of variables for satisfying the scaling criteria by relaxing different scaling requirements. For example, the vertical geometry scale is relaxed to satisfy the viscous and gravitational forces using the concept of the same fluid and porous medium. Selecting the appropriate approach to use is largely 
dependent on the specific process being modelled. Two main factors must be considered in order to choose a proper approach. First, the selection of a major mechanism should be correctly scaled. Second, a minor mechanism should not have any significant effects on the selected approach. The best way to select a suitable approach is the comparison of different approaches. This comparison indicates which mechanism is scaled and which are not with an order of degree. This study will help to select an appropriate steam flooding technique with the minimum number of most influential dimensionless scaling groups.

Acknowledgements The authors would like to thank Research \& Development Corporation of Newfoundland and Labrador (RDC), Funding No. 210992, and Statoil Canada Ltd., Funding No. 211162, for providing financial support to accomplish this research under Statoil Chair in Reservoir Engineering at the Memorial University of Newfoundland, St. John's, NL, Canada.
Open Access This article is licensed under a Creative Commons Attribution 4.0 International License, which permits use, sharing, adaptation, distribution and reproduction in any medium or format, as long as you give appropriate credit to the original author(s) and the source, provide a link to the Creative Commons licence, and indicate if changes were made. The images or other third party material in this article are included in the article's Creative Commons licence, unless indicated otherwise in a credit line to the material. If material is not included in the article's Creative Commons licence and your intended use is not permitted by statutory regulation or exceeds the permitted use, you will need to obtain permission directly from the copyright holder. To view a copy of this licence, visit http://creativecommons.org/licenses/by/4.0/.

\section{Appendix}

See Table 3.
Table 3 Influence of different dimensionless groups on each approach

\begin{tabular}{|c|c|c|c|c|c|c|c|c|c|c|c|}
\hline \multirow[t]{2}{*}{ Dimensionless groups } & \multicolumn{5}{|c|}{ Approaches } & \multirow[t]{2}{*}{ Dimensionless groups } & \multicolumn{5}{|c|}{ Approaches } \\
\hline & 1 & 2 & 3 & 4 & 5 & & 1 & 2 & 3 & 4 & 5 \\
\hline$\pi_{1}=\frac{t_{R} u_{x w R}}{\phi x_{R} s_{w R}}$ & $x$ & $\downarrow a$ & $\sqrt{ }$ & $\sqrt{ }$ & $x$ & $\pi_{89}=\frac{\eta_{w R} p_{w R}}{\Gamma(1-\alpha) t_{R}^{\alpha-1} \phi_{R} s_{w R}^{2} z_{R}^{2}}$ & $x$ & $\sqrt{ }$ & $\sqrt{ }$ & $\sqrt{ }$ & $x$ \\
\hline$\pi_{2}=\frac{t_{R} u_{z w R}}{\phi z_{R} s_{w R}}$ & $x$ & $\downarrow a$ & $\sqrt{ }$ & & $x$ & $\pi_{90}=\frac{\eta_{g R} P_{w R}}{\Gamma(1-\alpha) t_{R}^{\alpha-1} \phi_{R} s_{w R} z_{R}^{2}}$ & $x$ & $\sqrt{ }$ & $\sqrt{ }$ & $\sqrt{ }$ & $x$ \\
\hline$\pi_{3}=\frac{D_{L w 1 R^{2} t_{R}}}{x_{R}^{2} s_{w R}}$ & $x$ & $x$ & $x$ & $\sqrt{ }$ & $x$ & $\pi_{91}=\frac{f_{x o R} \eta_{o R} p_{w R}}{\Gamma(1-\alpha) t_{R}^{\alpha-1} \phi_{R} s_{w R} z_{R}^{2}}$ & $x$ & $\downarrow \boldsymbol{a}^{\frac{2}{\alpha-1}}$ & $\downarrow \boldsymbol{a}^{\frac{2}{\alpha-1}}$ & $\sqrt{ }$ & $x$ \\
\hline$\pi_{4}=\frac{D_{T_{w 1 R} t_{R}}}{z_{R}^{2} s_{w R}}$ & $x$ & $x$ & $\uparrow$ & $\sqrt{ }$ & $x$ & $\pi_{92}=\frac{f_{x o R} \eta_{w_{N R}} p_{w R}}{\Gamma(1-\alpha) t_{R}^{\alpha-1} \phi_{R} s_{w R} z_{R}^{2}}$ & $x$ & $\downarrow \boldsymbol{a}^{\frac{2}{\alpha-1}}$ & $\downarrow \boldsymbol{a}^{\frac{2}{\alpha-1}}$ & $\sqrt{ }$ & $x$ \\
\hline$\pi_{5}=\frac{D_{L w 3 R} t_{R}}{x_{R}^{2} s_{w R}}$ & $x$ & $x$ & $x$ & $\sqrt{ }$ & $x$ & $\pi_{93}=\frac{f_{x o R} \eta_{g R} p_{w R}}{\Gamma(1-\alpha) t_{R}^{\alpha-1} \phi_{R} s_{w R} z_{R}^{2}}$ & $x$ & $\downarrow \boldsymbol{a}^{\frac{2}{\alpha-1}}$ & $\downarrow \boldsymbol{a}^{\frac{2}{\alpha-1}}$ & $\sqrt{ }$ & $x$ \\
\hline$\pi_{6}=\frac{D_{T_{w 33} t_{R}}}{z_{R}^{2} s_{w R}}$ & $x$ & $x$ & $\uparrow$ & $\sqrt{ }$ & $x$ & $\pi_{94}=\frac{f_{x w R} \eta_{o R} p_{w R}}{\Gamma(1-\alpha) t_{R}^{\alpha-1} \phi_{R} s_{w R}^{2} z_{R}^{2}}$ & $x$ & $\downarrow \boldsymbol{a}^{\frac{2}{\alpha-1}}$ & $\downarrow \boldsymbol{a}^{\frac{2}{\alpha-1}}$ & $\sqrt{ }$ & $x$ \\
\hline$\pi_{7}=\frac{D_{L w 4 R} t_{R}}{x_{R}^{2} s_{w R}}$ & $x$ & $x$ & $x$ & $\sqrt{ }$ & $x$ & $\pi_{95}=\frac{f_{x w R} \eta_{w R} p_{w R}}{\Gamma(1-\alpha) t_{R}^{\alpha-1} \phi_{R} s_{w R}^{2} z_{R}^{2}}$ & $x$ & $\downarrow \boldsymbol{a}^{\frac{2}{\alpha-1}}$ & $\downarrow \boldsymbol{a}^{\frac{2}{\alpha-1}}$ & $\sqrt{ }$ & $x$ \\
\hline$\pi_{8}=\frac{D_{T_{w A R} t_{R}}}{z_{R}^{2} s_{w R}}$ & $x$ & $x$ & $\uparrow$ & $\sqrt{ }$ & $x$ & $\pi_{96}=\frac{f_{x w R} \eta_{g_{R}} p_{w R}}{\Gamma(1-\alpha) t_{R}^{g-1} \phi_{R} s_{w R} z_{R}^{2}}$ & $x$ & $\downarrow \boldsymbol{a}^{\frac{2}{\alpha-1}}$ & $\downarrow \boldsymbol{a}^{\frac{2}{\alpha-1}}$ & $\sqrt{ }$ & $x$ \\
\hline$\pi_{9}=\frac{t_{R} u_{x o R}}{\phi x_{R} s_{o R}}$ & $x$ & $\downarrow a$ & $\sqrt{ }$ & $\sqrt{ }$ & $x$ & $\pi_{97}=\frac{\eta_{o R} p_{c o w R}}{\Gamma(1-\alpha) t_{R}^{\alpha-1} \phi_{R} s_{w R}^{2} z_{R}^{2}}$ & $x$ & $\sqrt{ }$ & $\sqrt{ }$ & $\sqrt{ }$ & $x$ \\
\hline$\pi_{10}=\frac{t_{R} u_{z o R}}{\phi z_{R} s_{o R}}$ & $x$ & $\downarrow a$ & $\sqrt{ }$ & $\sqrt{ }$ & $x$ & $\pi_{98}=\frac{\eta_{g R} p_{\text {cowR }}}{\Gamma(1-\alpha) t_{R}^{\alpha-1} \phi_{R} s_{w R} z_{R}^{2}}$ & $x$ & $\sqrt{ }$ & $\sqrt{ }$ & $\sqrt{ }$ & $x$ \\
\hline$\pi_{11}=\frac{D_{L O 1 R} t_{R}}{x_{R}^{2} s_{o R}}$ & $x$ & $x$ & $x$ & $\sqrt{ }$ & $x$ & $\pi_{99}=\frac{f_{x o R} \eta_{o R} p_{c o w R}}{\Gamma(1-\alpha) t_{R}^{-1} \phi_{R} s_{w R} z_{R}^{2}}$ & $x$ & $\sqrt{ }$ & $\sqrt{ }$ & $\sqrt{ }$ & $x$ \\
\hline$\pi_{12}=\frac{D_{T o 1 R} t_{R}}{z_{R}^{2} s_{o R}}$ & $x$ & $x$ & $\uparrow$ & $\sqrt{ }$ & $\times$ & $\pi_{100}=\frac{f_{x o R} \eta_{g R} p_{c o w R}}{\Gamma(1-\alpha) t_{R}^{\alpha-1} \phi_{R} s_{w R} z_{R}^{2}}$ & $x$ & $\sqrt{ }$ & $\sqrt{ }$ & $\sqrt{ }$ & $x$ \\
\hline$\pi_{13}=\frac{D_{L o 2 R} t_{R}}{x_{R}^{2} s_{o R}}$ & $x$ & $x$ & $x$ & $\sqrt{ }$ & $x$ & $\pi_{101}=\frac{f_{x w R} \eta_{o R} p_{c o w R}}{\Gamma(1-\alpha) t_{R}^{\alpha-1} \phi_{R} s_{w R} z_{R}^{2}}$ & $x$ & $\sqrt{ }$ & $\sqrt{ }$ & $\sqrt{ }$ & $x$ \\
\hline$\pi_{14}=\frac{D_{T_{o 2 R} t_{R}}}{z_{R}^{2} s_{o R}}$ & $x$ & $x$ & $\uparrow$ & $\sqrt{ }$ & $x$ & $\pi_{102}=\frac{f_{x w R} \eta_{o_{0 R}} p_{c o w R}}{\Gamma(1-\alpha) t_{R}^{\alpha-1} \phi_{R} s_{w R} z_{R}^{2}}$ & $x$ & $\sqrt{ }$ & $\sqrt{ }$ & $\sqrt{ }$ & $x$ \\
\hline$\pi_{15}=\frac{D_{L o 4 R} t_{R}}{x_{R}^{2} s_{o R}}$ & $x$ & $x$ & $x$ & $\sqrt{ }$ & $x$ & $\pi_{103}=\frac{\eta_{g R} p_{c g o R}}{\Gamma(1-\alpha) t_{R}^{t^{-1}} \phi_{R} s_{w R} z_{R}^{2}}$ & $x$ & $\sqrt{ }$ & $\sqrt{ }$ & $\sqrt{ }$ & $x$ \\
\hline$\pi_{16}=\frac{D_{T o 4 R^{2} t_{R}}}{z_{R}^{2} s_{o R}}$ & $x$ & $x$ & $\uparrow$ & $\sqrt{ }$ & $x$ & $\pi_{104}=\frac{f_{x o R} \eta_{g R} p_{c g o R}}{\Gamma(1-\alpha) t_{R}^{\alpha-1} \phi_{R} s_{w R} z_{R}^{2}}$ & $x$ & $\sqrt{ }$ & $\sqrt{ }$ & $\sqrt{ }$ & $x$ \\
\hline$\pi_{17}=\frac{u_{x g R} t_{R}}{\phi x_{R} s_{g R}}$ & $x$ & $\downarrow a$ & $\sqrt{ }$ & $\sqrt{ }$ & $x$ & $\pi_{105}=\frac{f_{x w R} \eta_{g R} p_{c g o R}}{\Gamma(1-\alpha) t_{R}^{\alpha-1} \phi_{R} s_{w R} z_{R}^{2}}$ & $x$ & $\sqrt{ }$ & $\sqrt{ }$ & $\sqrt{ }$ & $x$ \\
\hline$\pi_{18}=\frac{u_{z g R} t_{R}}{\phi z_{R} s_{g R}}$ & $x$ & $\downarrow a$ & $\sqrt{ }$ & $\sqrt{ }$ & $x$ & $\pi_{106}=\frac{t_{R} D_{L w 1 R}}{s_{w R} x_{R}^{2}}$ & $x$ & $x$ & $x$ & $\sqrt{ }$ & $x$ \\
\hline$\pi_{19}=\frac{u_{x o R}}{U_{x R}}$ & $\sqrt{ }$ & $\downarrow a$ & $\sqrt{ }$ & $\sqrt{ }$ & $\sqrt{ }$ & $\pi_{107}=\frac{t_{R} D_{L w 3 R}}{s_{w R} x_{R}^{2}}$ & $x$ & $x$ & $x$ & $\sqrt{ }$ & $x$ \\
\hline$\pi_{20}=\frac{u_{x w R}}{U_{x R}}$ & $\sqrt{ }$ & $\downarrow a$ & $\sqrt{ }$ & $\sqrt{ }$ & $\sqrt{ }$ & $\pi_{108}=\frac{t_{R} D_{L w 4 R}}{s_{w R} x_{R}^{2}} \times$ & & $x$ & $x$ & $\sqrt{ }$ & $x$ \\
\hline$\pi_{21}=\frac{u_{x g R}}{U_{x R}}$ & $\sqrt{ }$ & $\downarrow a$ & $\sqrt{ }$ & $\sqrt{ }$ & $\sqrt{ }$ & $\pi_{109}=\frac{t_{R} D_{T w 1 R}}{s_{w R} z_{R}^{2}}$ & $x$ & $\times$ & $\uparrow$ & $\sqrt{ }$ & $x$ \\
\hline$\pi_{22}=\frac{u_{z o R}}{U_{z R}}$ & $\sqrt{ }$ & $\downarrow a$ & $\sqrt{ }$ & $\sqrt{ }$ & $\sqrt{ }$ & $\pi_{110}=\frac{t_{R} D_{T_{w 3 R}}}{s_{w R} z_{R}^{2}}$ & $x$ & $x$ & $\uparrow$ & $\sqrt{ }$ & $x$ \\
\hline
\end{tabular}


Table 3 (continued)

\begin{tabular}{|c|c|c|c|c|c|c|c|c|c|c|c|}
\hline \multirow[t]{2}{*}{ Dimensionless groups } & \multicolumn{5}{|c|}{ Approaches } & \multirow[t]{2}{*}{ Dimensionless groups } & \multicolumn{5}{|c|}{ Approaches } \\
\hline & 1 & 2 & 3 & 4 & 5 & & 1 & 2 & 3 & 4 & 5 \\
\hline$=\frac{u_{z N R}}{U_{-R}}$ & $\sqrt{ }$ & $\downarrow a$ & $\sqrt{ }$ & $\sqrt{ }$ & $\sqrt{ }$ & $\pi_{111}=\frac{t_{R}}{s}$ & $x$ & $x$ & $\bar{\uparrow}$ & $\sqrt{ }$ & $x$ \\
\hline${ }_{4}=\frac{u_{z g R}}{U_{z R}}$ & $\sqrt{ }$ & $\downarrow a$ & $\sqrt{ }$ & $\sqrt{ }$ & $\sqrt{ }$ & $\pi_{112}=\frac{k_{w w R} \lambda_{x w R} p_{w R R}}{x_{p} u_{u_{w w}}}$ & $\uparrow a$ & $\sqrt{ }$ & $\sqrt{ }$ & $\sqrt{ }$ & $\uparrow a$ \\
\hline${ }_{5}=\frac{u_{x_{0 O R}}}{f_{x o R} U_{X R}}$ & $\sqrt{ }$ & $\sqrt{ }$ & $\sqrt{ }$ & $\sqrt{ }$ & $\sqrt{ }$ & 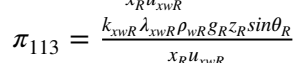 & $\uparrow a$ & $x$ & $\sqrt{ }$ & $x$ & $\uparrow a$ \\
\hline${ }_{26}=\frac{u_{z o R}}{f_{z o R} U_{v R}}$ & $\sqrt{ }$ & $\sqrt{ }$ & $\sqrt{ }$ & $\sqrt{ }$ & $\sqrt{ }$ & $\pi_{114}=\frac{k_{x o R} \lambda_{x o R} P_{O R}}{x_{0,} u_{x p}}$ & $\uparrow a$ & $\sqrt{ }$ & $\sqrt{ }$ & $\sqrt{ }$ & $\uparrow a$ \\
\hline $27=\frac{u_{x w R}}{f_{x w R} U_{x R}}$ & $\sqrt{ }$ & $\sqrt{ }$ & $\sqrt{ }$ & $\sqrt{ }$ & $\sqrt{ }$ & 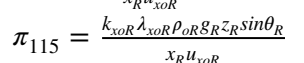 & $\uparrow a$ & $x$ & $\sqrt{ }$ & $x$ & $\uparrow a$ \\
\hline$\pi_{28}=\frac{u_{z w R}}{f_{z w R} U_{z R}}$ & $\sqrt{ }$ & $\sqrt{ }$ & $\sqrt{ }$ & $\sqrt{ }$ & $\sqrt{ }$ & $\pi_{116}=\frac{k_{x g R} \lambda_{x g R} P_{g R}}{x_{R} u_{x g R}}$ & $\uparrow a$ & $\sqrt{ }$ & $\sqrt{ }$ & $\sqrt{ }$ & $\uparrow a$ \\
\hline$=\frac{u_{x g R}}{f_{x g R} U_{x R}}$ & $\sqrt{ }$ & $\sqrt{ }$ & $\sqrt{ }$ & $\sqrt{ }$ & $\sqrt{ }$ & $\pi_{117}=\frac{k_{x g R} \lambda_{x g R} \rho_{g R} g_{R} z_{R} \sin \theta_{R}}{x_{R} u_{x g R}}$ & $\uparrow a$ & $x$ & $\sqrt{ }$ & $x$ & $\uparrow a$ \\
\hline$=\frac{f_{x o R}}{f_{x x R}}$ & $\sqrt{ }$ & $\sqrt{ }$ & $\sqrt{ }$ & $\sqrt{ }$ & $\sqrt{ }$ & 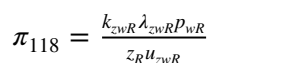 & $\uparrow a$ & $\sqrt{ }$ & $\sqrt{ }$ & $\sqrt{ }$ & $\uparrow a$ \\
\hline$=\frac{f_{\text {surR }}}{f_{x g R}}$ & $\sqrt{ }$ & $\sqrt{ }$ & $\sqrt{ }$ & $\sqrt{ }$ & $\sqrt{ }$ & $\pi_{119}=\frac{k_{z w R} \lambda_{z w R} \lambda_{w W R} \rho_{w h} g_{R} z_{R} \sin \theta_{R}}{z_{R} u_{w w R}}$ & $\uparrow a$ & $x$ & $\sqrt{ }$ & $x$ & $\uparrow a$ \\
\hline $2=\frac{u_{z Z R}}{f_{z Z R} U_{z R}}$ & $\sqrt{ }$ & $\sqrt{ }$ & $\sqrt{ }$ & $\sqrt{ }$ & $\sqrt{ }$ & $\pi_{120}=\frac{k_{z o R} \lambda_{z o R} P_{O R}}{z_{R} u_{z O R}}$ & $\uparrow a$ & $\sqrt{ }$ & $\sqrt{ }$ & $\sqrt{ }$ & $\uparrow a$ \\
\hline $33=\frac{f_{z O R}}{c}$ & $\sqrt{ }$ & $\sqrt{ }$ & $\sqrt{ }$ & $\sqrt{ }$ & $\sqrt{ }$ & $\pi_{121}=\frac{k_{z o R} \lambda_{z o R} \rho_{o R} g_{R} z_{R R} \sin \theta_{R}}{z_{R} R_{z o R}}$ & $\uparrow a$ & $x$ & $\sqrt{ }$ & $x$ & $\uparrow a$ \\
\hline$f_{z w R}$ & $\sqrt{ }$ & $\sqrt{ }$ & $\sqrt{ }$ & $\sqrt{ }$ & $\sqrt{ }$ & 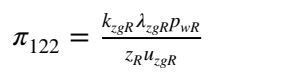 & $\uparrow a$ & $x$ & $\sqrt{ }$ & $\sqrt{ }$ & $\uparrow a$ \\
\hline $35=\frac{\eta_{o \alpha} p_{W R}}{\Gamma(1-\alpha)_{R}^{R-1} \phi_{R} s_{W R} x_{R}^{2}}$ & $x$ & $\sqrt{ }$ & $\sqrt{ }$ & $\sqrt{ }$ & $x$ & $\pi_{123}=\frac{k_{z g R} \lambda_{z g R} \rho_{g R} g_{R} z_{R} \sin \theta_{R}}{z_{R} u_{z R R}}$ & $\uparrow a$ & $x$ & $\sqrt{ }$ & $x$ & $\uparrow a$ \\
\hline${ }_{6}=\frac{\eta_{w R p_{w R}}}{\Gamma(1-\alpha) t_{R}^{\alpha-1} \phi_{R} s_{W R} x_{R}^{2}}$ & $x$ & $\sqrt{ }$ & $\sqrt{ }$ & $\sqrt{ }$ & $x$ & $\pi_{124}=\frac{\sigma_{\text {owR }} J_{R}\left(s_{w}\right) \cos \theta_{R} \sqrt{\frac{\phi_{R}}{k_{R}}}}{p_{\text {cowr }}}$ & $x$ & $\sqrt{ }$ & $x$ & $x$ & $x$ \\
\hline$=\frac{\eta_{g R} p_{w R}}{\Gamma(1-\alpha) t_{R}^{\alpha-1} \phi_{R} s_{w R} x_{R}^{2}}$ & $x$ & $\sqrt{ }$ & $\sqrt{ }$ & $\sqrt{ }$ & $x$ & $\pi_{125}=\frac{\sigma_{g o R} J_{R}\left(s_{g}\right) \cos \theta_{R} \sqrt{\frac{\phi_{R}}{k_{R}}}}{p_{c g o R}}$ & $x$ & $\sqrt{ }$ & $x$ & $x$ & $x$ \\
\hline 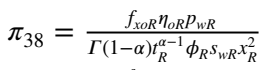 & $x$ & $\downarrow \frac{2}{a-1}$ & $\downarrow a^{\frac{2}{\alpha-1}}$ & $\sqrt{ }$ & $x$ & $\pi_{126}=\frac{s_{o R}}{s_{w R}}$ & $x$ & $\sqrt{ }$ & $\sqrt{ }$ & $\sqrt{ }$ & $x$ \\
\hline$\pi_{39}=\frac{f_{x o R} \eta_{w W} P_{w R}}{\Gamma(1-\alpha) t_{R}^{t-1} \phi_{R} s_{w R} x_{R}^{2}}$ & $x$ & $\downarrow \frac{2}{\alpha-1}$ & $\downarrow a^{\frac{2}{\alpha-1}}$ & $\sqrt{ }$ & $x$ & $\pi_{127}=\frac{s_{g R}}{s_{w R}}$ & $x$ & $\sqrt{ }$ & $\sqrt{ }$ & $\sqrt{ }$ & $x$ \\
\hline$\pi_{40}=\frac{f_{x o R} \eta_{R R} p_{w R}}{\Gamma(1-\alpha)_{R}^{\alpha-1} \phi_{R} s_{w R}^{2} x_{R}^{2}}$ & $x$ & $\downarrow \boldsymbol{a}^{\frac{2}{\alpha-1}}$ & $\downarrow \boldsymbol{a}^{\frac{2}{\alpha-1}}$ & $\sqrt{ }$ & $x$ & $\pi_{128}=\frac{s_{o}-s_{o r}}{1-s_{w i}-S_{o r}}$ & $x$ & $\sqrt{ }$ & $\sqrt{ }$ & $\sqrt{ }$ & $x$ \\
\hline$\pi_{41}=\frac{f_{x w R} \eta_{o R} p_{w R}}{\Gamma(1-\alpha) t_{R}^{\alpha-1} \phi_{R} s_{w R} x_{R}^{2}}$ & $x$ & $\downarrow \frac{2}{\alpha-1}$ & $\downarrow \boldsymbol{a}^{\frac{2}{\alpha-1}}$ & $\sqrt{ }$ & $x$ & $\pi_{129}=\frac{s_{w}-s_{w i}}{1-s_{w i}-s_{o r}}$ & $x$ & $\sqrt{ }$ & $\sqrt{ }$ & $\sqrt{ }$ & $x$ \\
\hline $42=\frac{f_{w w R} \eta_{w W-} p_{w R}}{\Gamma(1-\alpha)_{R}^{w_{R}-1} \phi_{R} s_{w R} x_{R}^{2}}$ & $x$ & $\downarrow \frac{2}{\alpha-1}$ & $\downarrow a^{\frac{2}{\alpha-1}}$ & $\sqrt{ }$ & $x$ & $\pi_{130}=\frac{s_{g}-s_{g c}}{1-s_{w i}-s_{o r}}$ & $x$ & $\sqrt{ }$ & $\sqrt{ }$ & $\sqrt{ }$ & $x$ \\
\hline$\pi_{43}=\frac{f_{x w R} \eta_{g R} p_{w R}}{\Gamma(1-\alpha) t_{R}^{\alpha-1} \phi_{R} s_{W R} x_{R}^{2}}$ & $x$ & $\downarrow \boldsymbol{a}^{\frac{2}{\alpha-1}}$ & $\downarrow \boldsymbol{a}^{\frac{2}{\alpha-1}}$ & $\sqrt{ }$ & $x$ & $\pi_{131}=\frac{U_{t} t}{L \phi\left(1-s_{w i}-s_{o r}\right)}$ & $\downarrow a^{2}$ & $\downarrow a^{2}$ & $\downarrow a^{2}$ & $\downarrow a^{2}$ & $\downarrow a^{2}$ \\
\hline$\pi_{44}=\frac{\eta_{o o} p_{c o w R}}{\Gamma(1-\alpha) t_{R}^{\sigma-1} \phi_{R} S_{w R} x_{R}^{2}}$ & $x$ & $\sqrt{ }$ & $\sqrt{ }$ & $\sqrt{ }$ & $x$ & $\pi_{132}=\frac{s_{w i}}{s_{w D}}$ & $x$ & $\sqrt{ }$ & $\sqrt{ }$ & $\sqrt{ }$ & $x$ \\
\hline$\pi_{45}=\frac{\eta_{g R} p_{c o w R}}{\Gamma(1-\alpha) t_{R}^{t-1} \phi_{R} s_{w R} x_{R}^{2}}$ & $x$ & $\sqrt{ }$ & $\sqrt{ }$ & $\sqrt{ }$ & $x$ & $\pi_{133}=\frac{s_{g i}}{s_{g R}}$ & $x$ & $\sqrt{ }$ & $\sqrt{ }$ & $\sqrt{ }$ & $x$ \\
\hline$\pi_{46}=\frac{f_{x o R} \eta_{0 R} p_{c o w R}}{\Gamma(1-\alpha) t_{R}^{\alpha-1} \phi_{R} s_{w R} x_{R}^{2}}$ & $x$ & $\sqrt{ }$ & $\sqrt{ }$ & $\sqrt{ }$ & $x$ & $\pi_{134}=\frac{s_{o i}}{s_{o R}}$ & $x$ & $\sqrt{ }$ & $\sqrt{ }$ & $\sqrt{ }$ & $x$ \\
\hline$\pi_{47}=\frac{f_{x \sim R} n_{g R} p_{c o w R}}{\Gamma(1-\alpha) t_{R}^{\alpha-1} \phi_{R} s_{w R} x_{R}^{2}}$ & $x$ & $\sqrt{ }$ & $\sqrt{ }$ & $\sqrt{ }$ & $x$ & $\pi_{135}=\frac{E_{2 i}}{E_{o 2 R}}$ & $x$ & $x$ & $x$ & $\sqrt{ }$ & $\sqrt{ }$ \\
\hline$\pi_{48}=\frac{f_{x w R} \eta_{n_{R}} p_{c o w R}}{\Gamma(1-\alpha) t_{R}^{t-1} \phi_{R} s_{w R} x_{R}^{2}}$ & $x$ & $\sqrt{ }$ & $\sqrt{ }$ & $\sqrt{ }$ & $x$ & $\pi_{136}=\frac{E_{3 i}}{E_{w 3 R}}$ & $x$ & $x$ & $x$ & $\sqrt{ }$ & $\sqrt{ }$ \\
\hline$\pi_{49}=\frac{f_{w w R} \eta_{o R} p_{c o w R}}{\Gamma(1-\alpha) t_{R}^{\alpha-1} \phi_{R} s_{W R} x_{R}^{2}}$ & $x$ & $\sqrt{ }$ & $\sqrt{ }$ & $\sqrt{ }$ & $x$ & $\pi_{137}=\frac{E_{1 j}}{E_{g 1 R}}$ & $x$ & $x$ & $x$ & $\sqrt{ }$ & $\sqrt{ }$ \\
\hline$\pi_{50}=\frac{\eta_{g R} p_{c g o R}}{\Gamma(1-\alpha) t_{R}^{t-1} \phi_{R} s_{w R} x_{R}^{2}}$ & $x$ & $\sqrt{ }$ & $\sqrt{ }$ & $\sqrt{ }$ & $x$ & $\pi_{138}=\frac{E_{4 j}}{E_{w 4 R}}$ & $x$ & $x$ & $x$ & $\sqrt{ }$ & $\sqrt{ }$ \\
\hline$\pi_{51}=\frac{f_{x o R} \eta_{g R} p_{c o o R}}{\Gamma(1-\alpha) t_{R}^{\alpha-1} \phi_{R} s_{W R} x_{R}^{2}}$ & $x$ & $\sqrt{ }$ & $\sqrt{ }$ & $\sqrt{ }$ & $x$ & $\pi_{139}=\frac{\Delta p_{g R}}{p_{g R}}$ & $x$ & $\sqrt{ }$ & $\sqrt{ }$ & $\sqrt{ }$ & $\sqrt{ }$ \\
\hline 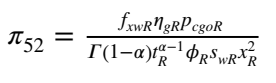 & $x$ & $\sqrt{ }$ & $\sqrt{ }$ & $\sqrt{ }$ & $x$ & $\pi_{140}=\frac{\rho_{g} g_{R} H_{R}}{P_{g R}}$ & $\sqrt{ }$ & $x$ & $\sqrt{ }$ & $x$ & $\sqrt{ }$ \\
\hline$\pi_{53}=\frac{p_{w R}}{p_{\text {cowR }}}$ & $x$ & $\sqrt{ }$ & $\sqrt{ }$ & $\sqrt{ }$ & $\sqrt{ }$ & $\pi_{141}=\frac{\rho_{g R} g_{R} z_{R}}{p_{g R}}$ & $\sqrt{ }$ & $x$ & $\sqrt{ }$ & $x$ & $\sqrt{ }$ \\
\hline
\end{tabular}


Table 3 (continued)

\begin{tabular}{|c|c|c|c|c|c|c|c|c|c|c|c|}
\hline \multirow[t]{2}{*}{ Dimensionless groups } & \multicolumn{5}{|c|}{ Approaches } & \multirow[t]{2}{*}{ Dimensionless groups } & \multicolumn{5}{|c|}{ Approaches } \\
\hline & 1 & 2 & 3 & 4 & 5 & & 1 & 2 & 3 & 4 & 5 \\
\hline$\overline{\pi_{54}=\frac{x_{R}^{2}}{z_{R}^{2}}}$ & $\downarrow a$ & $\downarrow a$ & $\downarrow a$ & $\downarrow a$ & $\downarrow a$ & $\pi_{142}=\frac{\Delta p_{o R}}{p_{o R}}$ & $x$ & $\sqrt{ }$ & $\sqrt{ }$ & $\sqrt{ }$ & $\sqrt{ }$ \\
\hline$\pi_{55}=\frac{p_{w R} x_{R}^{2}}{p_{c o w R} z_{R}^{2}}$ & $x$ & $\sqrt{ }$ & $\sqrt{ }$ & $\sqrt{ }$ & $\sqrt{ }$ & $\pi_{143}=\frac{\rho_{o R} g_{R} H_{R}}{p_{o R}}$ & $\sqrt{ }$ & $x$ & $\sqrt{ }$ & $x$ & $\sqrt{ }$ \\
\hline$\pi_{56}=\frac{p_{w R}}{p_{c g o R}}$ & $x$ & $\sqrt{ }$ & $\sqrt{ }$ & $\sqrt{ }$ & $\sqrt{ }$ & $\pi_{144}=\frac{\rho_{o R} g_{R} z_{R}}{p_{o R}}$ & $\sqrt{ }$ & $x$ & $\sqrt{ }$ & $x$ & $\sqrt{ }$ \\
\hline$\pi_{57}=\frac{p_{w R} x_{R}^{2}}{p_{c g o R} z_{R}^{2}}$ & $x$ & $\sqrt{ }$ & $\sqrt{ }$ & $\sqrt{ }$ & $\sqrt{ }$ & $\pi_{145}=\frac{\Delta p_{w R}}{p_{w R}}$ & $\sqrt{ }$ & $\sqrt{ }$ & $\sqrt{ }$ & $\sqrt{ }$ & $\sqrt{ }$ \\
\hline$\pi_{58}=\frac{p_{\text {cow } R}}{p_{c g o R}}$ & $x$ & $\sqrt{ }$ & $\sqrt{ }$ & $\sqrt{ }$ & $\sqrt{ }$ & $\pi_{146}=\frac{\rho_{w R} g_{R} H_{R}}{p_{w R}}$ & $\sqrt{ }$ & $x$ & $\sqrt{ }$ & $x$ & $\sqrt{ }$ \\
\hline$\pi_{59}=\frac{p_{\text {cowR }} x_{R}^{2}}{p_{\text {cgoR }} z_{R}^{2}}$ & $x$ & $\sqrt{ }$ & $\sqrt{ }$ & $\sqrt{ }$ & $\sqrt{ }$ & $\pi_{147}=\frac{\rho_{w R} g_{R} z_{R}}{p_{w R}}$ & $\sqrt{ }$ & $x$ & $\sqrt{ }$ & $x$ & $\sqrt{ }$ \\
\hline$\pi_{60}=\frac{f_{x o R} p_{w R}}{p_{c o w R}}$ & $x$ & $\sqrt{ }$ & $\sqrt{ }$ & $\sqrt{ }$ & $\sqrt{ }$ & $\pi_{148}=\frac{H U_{t}}{u_{g x R} x_{R}}$ & $\sqrt{ }$ & $\sqrt{ }$ & $\sqrt{ }$ & $\sqrt{ }$ & $\sqrt{ }$ \\
\hline$\pi_{61}=\frac{f_{x o R} x_{R}^{2}}{z_{R}^{2}}$ & $\downarrow a$ & $\sqrt{ }$ & $\sqrt{ }$ & $\sqrt{ }$ & $\sqrt{ }$ & $\pi_{149}=\frac{z_{R}^{2} D_{L o 1 R}}{x_{R}^{2} D_{T o 1 R}}$ & $x$ & $x$ & $x$ & $\sqrt{ }$ & $x$ \\
\hline$\pi_{62}=\frac{f_{x o R} p_{w R} x_{R}^{2}}{p_{c o w R} z_{R}^{2}}$ & $x$ & $\sqrt{ }$ & $\sqrt{ }$ & $\sqrt{ }$ & $\sqrt{ }$ & $\pi_{150}=\frac{z_{R}^{2} D_{L o 2 R}}{x_{R}^{2} D_{T o 2 R}}$ & $x$ & $x$ & $x$ & $\sqrt{ }$ & $x$ \\
\hline$\pi_{63}=\frac{f_{x o R} p_{w R}}{p_{c g o R}}$ & $x$ & $\sqrt{ }$ & $\sqrt{ }$ & $\sqrt{ }$ & $\sqrt{ }$ & $\pi_{151}=\frac{z_{R}^{2} D_{L o 4 R}}{x_{R}^{2} D_{T o 4 R}}$ & $x$ & $x$ & $x$ & $\sqrt{ }$ & $x$ \\
\hline$\pi_{64}=\frac{f_{x o R} p_{w R} x_{R}^{2}}{p_{c g o R} z_{R}^{2}}$ & $\times$ & $\sqrt{ }$ & $\sqrt{ }$ & $\sqrt{ }$ & $\sqrt{ }$ & $\pi_{152}=\frac{z_{R}^{2} D_{L w 1 R}}{x_{R}^{2} D_{T w 1 R}}$ & $x$ & $x$ & $x$ & $\sqrt{ }$ & $x$ \\
\hline$\pi_{65}=\frac{f_{\text {xoR }} p_{\text {cowR }}}{p_{c \text { cooR }}}$ & $x$ & $\sqrt{ }$ & $\sqrt{ }$ & $\sqrt{ }$ & $\sqrt{ }$ & $\pi_{153}=\frac{z_{R}^{2} D_{L w 2 R}}{x_{R}^{2} D_{T w 2 R}}$ & $x$ & $x$ & $x$ & $\sqrt{ }$ & $x$ \\
\hline$\pi_{66}=\frac{f_{x o R} p_{\text {cow } R} x_{R}^{2}}{p_{\text {cgoR }} z_{R}^{2}}$ & $x$ & $\sqrt{ }$ & $\sqrt{ }$ & $\sqrt{ }$ & $\sqrt{ }$ & $\pi_{154}=\frac{z_{R}^{2} D_{L w 4 R}}{x_{R}^{2} D_{T w 4 R}}$ & $x$ & $x$ & $x$ & $\sqrt{ }$ & $x$ \\
\hline$\pi_{67}=\frac{f_{x w R} p_{w R}}{p_{c o w R}}$ & $x$ & $\sqrt{ }$ & $\sqrt{ }$ & $\sqrt{ }$ & $\sqrt{ }$ & $\pi_{155}=\frac{H}{z_{R}}$ & $\downarrow a$ & $\downarrow a$ & $\downarrow a$ & $\downarrow a$ & $\downarrow a$ \\
\hline$\pi_{68}=\frac{f_{x w R} x_{R}^{2}}{z_{R}^{2}}$ & $\downarrow a$ & $\sqrt{ }$ & $\sqrt{ }$ & $\sqrt{ }$ & $\sqrt{ }$ & $\pi_{156}=\frac{L}{x_{R}}$ & $\downarrow a$ & $\downarrow a$ & $\downarrow a$ & $\downarrow a$ & $\downarrow a$ \\
\hline$\pi_{69}=\frac{f_{x w R} p_{w R} x_{R}^{2}}{f_{x w R} p_{c o w R} z_{R}^{2}}$ & $x$ & $\sqrt{ }$ & $\sqrt{ }$ & $\sqrt{ }$ & $\sqrt{ }$ & $\pi_{157}=\frac{H U_{t}}{u_{g \times R} z_{R}}$ & $\sqrt{ }$ & $\sqrt{ }$ & $\sqrt{ }$ & $\sqrt{ }$ & $x$ \\
\hline$\pi_{70}=\frac{f_{x w R} p_{w R}}{p_{c g o R}}$ & $x$ & $\sqrt{ }$ & $\sqrt{ }$ & $\sqrt{ }$ & $\sqrt{ }$ & $\pi_{158}=\frac{K_{h r} T A t}{L \Delta T M_{1} V}$ & $x$ & $\sqrt{ }$ & $x$ & $\sqrt{ }$ & $x$ \\
\hline$\pi_{71}=\frac{f_{\text {wwR }} p_{w R} x_{R}^{2}}{p_{c g o R} z_{R}^{2}}$ & $x$ & $\sqrt{ }$ & $\sqrt{ }$ & $\sqrt{ }$ & $\sqrt{ }$ & $\pi_{159}=\frac{K_{h f} T A t}{L \Delta T M_{1} V}$ & $x$ & $\sqrt{ }$ & $x$ & $\sqrt{ }$ & $x$ \\
\hline$\pi_{72}=\frac{f_{x w R} p_{c o w R}}{p_{c g o R}}$ & $x$ & $\sqrt{ }$ & $\sqrt{ }$ & $\sqrt{ }$ & $\sqrt{ }$ & $\pi_{160}=\frac{t C_{p o} \rho_{o} u_{o x} A}{M_{1} V}$ & $x$ & $\sqrt{ }$ & $x$ & $\sqrt{ }$ & $x$ \\
\hline$\pi_{73}=\frac{f_{\text {xwR }} P_{\text {cow }} x_{R}^{2}}{p_{c g o R} z_{R}^{2}}$ & $x$ & $\sqrt{ }$ & $\sqrt{ }$ & $\sqrt{ }$ & $\sqrt{ }$ & $\pi_{161}=\frac{t C_{p w} \rho_{w} u_{w x} A}{M_{1} V}$ & $x$ & $\sqrt{ }$ & $x$ & $\sqrt{ }$ & $x$ \\
\hline$\pi_{74}=\frac{f_{z o R} p_{w R} x_{R}^{2}}{p_{c o w R} z_{R}^{2}}$ & $x$ & $\sqrt{ }$ & $\sqrt{ }$ & $\sqrt{ }$ & $\sqrt{ }$ & $\pi_{162}=\frac{t C_{p o} \rho_{o} \phi S_{o} v_{x} A}{M_{1} V}$ & $x$ & $\sqrt{ }$ & $x$ & $\sqrt{ }$ & $x$ \\
\hline$\pi_{75}=\frac{f_{z o R} p_{w R}}{p_{c g o R}}$ & $x$ & $\sqrt{ }$ & $\sqrt{ }$ & $\sqrt{ }$ & $\sqrt{ }$ & $\pi_{163}=\frac{t C_{p w} \rho_{w} \phi S_{w} v_{x} A}{M_{1} V}$ & $x$ & $\sqrt{ }$ & $x$ & $\sqrt{ }$ & $x$ \\
\hline$\pi_{76}=\frac{f_{z o R} p_{w R} x_{R}^{2}}{p_{c o w R} z_{R}^{2}}$ & $\times$ & $\sqrt{ }$ & $\sqrt{ }$ & $\sqrt{ }$ & $\sqrt{ }$ & $\pi_{164}=\frac{(1-\phi) C_{p r} t \rho_{r} v_{x} A}{\Delta T M_{1} V}$ & $x$ & $\sqrt{ }$ & $x$ & $\sqrt{ }$ & $x$ \\
\hline$\pi_{77}=\frac{f_{z o R} p_{w R}}{p_{c g o R}}$ & $x$ & $\sqrt{ }$ & $\sqrt{ }$ & $\sqrt{ }$ & $\sqrt{ }$ & $\pi_{165}=\frac{m_{s} f_{s} L_{v} t}{\Delta T M_{1} V}$ & $\times$ & $\sqrt{ }$ & $x$ & $\sqrt{ }$ & $x$ \\
\hline$\pi_{78}=\frac{f_{z o R} p_{w R} x_{R}^{2}}{p_{c g o R} z_{R}^{2}}$ & $x$ & $\sqrt{ }$ & $\sqrt{ }$ & $\sqrt{ }$ & $\sqrt{ }$ & $\pi_{166}=\frac{m_{s} C_{p w} t}{M_{1} V}$ & $x$ & $\sqrt{ }$ & $x$ & $\sqrt{ }$ & $x$ \\
\hline$\pi_{79}=\frac{f_{z o R} p_{\text {cow }}}{p_{\text {cgoR }}}$ & $x$ & $\sqrt{ }$ & $\sqrt{ }$ & $\sqrt{ }$ & $\sqrt{ }$ & $\pi_{167}=\frac{(1-\phi) \rho_{r} C_{p r}}{M_{1}}$ & $x$ & $\sqrt{ }$ & $x$ & $\sqrt{ }$ & $x$ \\
\hline$\pi_{80}=\frac{f_{x o R} p_{c o w R} x_{R}^{2}}{p_{c g o R} z_{R}^{2}}$ & $x$ & $\sqrt{ }$ & $\sqrt{ }$ & $\sqrt{ }$ & $\sqrt{ }$ & $\pi_{168}=\frac{\phi C_{p w} \rho_{w} S_{w}}{M_{1}}$ & $x$ & $\sqrt{ }$ & $x$ & $\sqrt{ }$ & $\times$ \\
\hline$\pi_{81}=\frac{f_{\text {swR }} p_{w R}}{p_{c o w R}}$ & $\times$ & $\sqrt{ }$ & $\sqrt{ }$ & $\sqrt{ }$ & $\sqrt{ }$ & $\pi_{169}=\frac{\phi c_{p o} \rho_{o} S_{0}}{M_{1}}$ & $x$ & $\sqrt{ }$ & $x$ & $\sqrt{ }$ & $x$ \\
\hline$\pi_{82}=\frac{f_{x w R} x_{R}^{2}}{z_{R}^{2}}$ & $x$ & $\sqrt{ }$ & $\sqrt{ }$ & $\sqrt{ }$ & $\sqrt{ }$ & $\pi_{170}=\frac{\phi C_{p g} \rho_{g} S_{g}}{M_{1}}$ & $x$ & $\sqrt{ }$ & $x$ & $\sqrt{ }$ & $\times$ \\
\hline
\end{tabular}


Table 3 (continued)

\begin{tabular}{|c|c|c|c|c|c|c|c|c|c|c|c|}
\hline \multirow[t]{2}{*}{ Dimensionless groups } & \multicolumn{5}{|c|}{ Approaches } & \multirow[t]{2}{*}{ Dimensionless groups } & \multicolumn{5}{|c|}{ Approaches } \\
\hline & 1 & 2 & 3 & 4 & 5 & & 1 & 2 & 3 & 4 & 5 \\
\hline$\pi_{83}=\frac{f_{x w R} p_{w R} x_{R}^{2}}{f_{x w R} p_{c o w R} z_{R}^{2}}$ & $x$ & $\sqrt{ }$ & $\sqrt{ }$ & $\sqrt{ }$ & $\sqrt{ }$ & $\pi_{171}=\frac{\phi L_{v} \rho_{g} S_{g}}{M_{1} \Delta T}$ & $x$ & $\sqrt{ }$ & $x$ & $\sqrt{ }$ & $x$ \\
\hline$\pi_{84}=\frac{f_{x w R} p_{w R}}{p_{c g o R}}$ & $x$ & $\sqrt{ }$ & $\sqrt{ }$ & $\sqrt{ }$ & $\sqrt{ }$ & $\pi_{172}=\frac{K_{h W S_{w}}}{K_{h f}}$ & $x$ & $\sqrt{ }$ & $\times$ & $\sqrt{ }$ & $x$ \\
\hline$\pi_{85}=\frac{f_{x w R} p_{w R} x_{R}^{2}}{p_{c g o R} z_{R}^{2}}$ & $x$ & $\sqrt{ }$ & $\sqrt{ }$ & $\sqrt{ }$ & $\sqrt{ }$ & $\pi_{173}=\frac{K_{h o} S_{o}}{K_{h f}}$ & $x$ & $\sqrt{ }$ & $x$ & $\sqrt{ }$ & $x$ \\
\hline$\pi_{86}=\frac{f_{x w R} p_{c o w R}}{p_{c g o R}}$ & $x$ & $\sqrt{ }$ & $\sqrt{ }$ & $\sqrt{ }$ & $\sqrt{ }$ & $\pi_{174}=\frac{K_{h g} S_{g}}{K_{h f}}$ & $x$ & $\sqrt{ }$ & $x$ & $\sqrt{ }$ & $x$ \\
\hline$\pi_{87}=\frac{f_{x w R} p_{c o w n} x_{R}^{2}}{p_{c g o R} z_{R}^{2}}$ & $x$ & $\sqrt{ }$ & $\sqrt{ }$ & $\sqrt{ }$ & $\sqrt{ }$ & $\pi_{175}=\frac{\phi K_{h f}}{K_{h e}}$ & $x$ & $\sqrt{ }$ & $\times$ & $\sqrt{ }$ & $x$ \\
\hline$\pi_{88}=\frac{\eta_{o R} p_{w R}}{\Gamma(1-\alpha) t_{R}^{\alpha-1} \phi_{R} s_{w R} z_{R}^{2}}$ & $x$ & $\sqrt{ }$ & $\sqrt{ }$ & $\sqrt{ }$ & $x$ & $\pi_{176}=\frac{(1-\phi) K_{h r}}{K_{h e}}$ & $x$ & $\sqrt{ }$ & $x$ & $\sqrt{ }$ & $x$ \\
\hline
\end{tabular}

$\sqrt{ }$ indicates the group is satisfied, $\times$ indicates the group is not satisfied, $\downarrow$ indicates the group is reduced, $\uparrow$ indicates the group is increased, $a$ indicate the dimension of scaling factor by which the model is reduced from prototype

\section{References}

Baker PE (1969) An experimental study of heat flow in steam flooding. Soc Petrol Eng J 9(01):89-99

Baker PE (1973) Effect of pressure and rate on steam zone development in steamflooding. Soc Petrol Eng J 13(05):274-284

Bear J (1972) Dynamics of fluids in porous media. American Elsevier, New York, p 764

Birkhoff GD (1950) Dynamical systems colloquium publications, vol 9, 2nd edn. American Mathematical Society, Providence

Bobek JE, Bail PT (1961) Model studies of oil displacement from thin sands by vertical water influx from adjacent shales. J Pet Technol 13(09):950-954

Bridgman PW (1931) Dimensional analysis, 2nd edn. Yale University Press, New Haven

Buckingham E (1914) On physically similar systems; illustrations of the use of dimensional equations. Phys Rev 4(4):345

Carpenter CW Jr, Bail PT, Bobek JE (1962) A verification of waterflood scaling in heterogeneous communicating flow models. Soc Pet Eng J 2(01):9-12. https://doi.org/10.2118/171-PA

Craig FF Jr, Geffen TM, Morse RA (1955) Oil recovery performance of pattern gas or water injection operations from model tests. Trans AIME 204:7-15

Craig FF, Sanderlin JL, Moore DW, Geffen TM (1957) A laboratory study of gravity segregation in frontal drives. Trans AIME 210:275

Ehrlich R, Hasiba HH, Raimondi P (1974) Alkaline waterflooding for wettability alteration-evaluating a potential field application. J Pet Technol 26(12):1-335. https://doi.org/10.2118/4905-PA

Engelberts WF, Klinkenberg LJ (1951) Laboratory experiments on the displacement of oil by water from packs of granular material. In: Paper no: WPC-4138, 3rd world petroleum congress, pp 544-554

Farouq Ali SM, Redford DA (1977) Physical modeling of in situ recovery methods for oil sands. Oil sands-Canada-Venezuela. CIMM, Montreal

Focken CM (1953) Dimensional Analysis and Their Application. Edward Arnold

Geertsma J, Croes GA, Schwarz N (1956) Theory of dimensionally scaled models of petroleum reservoirs. Trans AIME 207:118-127

Graham JW, Richardson JG (1959) Theory and application of imbibition phenomena in recovery of oil. J Pet Technol 11(02):65-69. https://doi.org/10.2118/1143-G
Henley DH, Owens WW, Craig FF Jr (1961) A scale-model study of bottom-water drives. J Pet Technol 13(01):90-98. https://doi. org/10.2118/1539-G-PA

Hossain ME (2017) Role of porosity on energy transport with equal rock-fluid temperatures during thermal EOR process. Arab J Sci Eng 42(4):1621-1631

Hossain ME, Mousavizadegan SH, Islam MR (2007) A novel memory based stress-strain model for reservoir characterization. J Nat Sci Sustain Technol 1(4):653-678

Hossain ME, Mousavizadegan SH, Islam MR (2008) A novel fluid flow model with memory for porous media application. In: Mechanics of time dependent material conference, march 30-April 4, 2008 , Monterey, California

Hossain ME, Mousavizadegan SH, Islam MR (2009) Effects of memory on the complex rock-fluid properties of a reservoir stressstrain model. Pet Sci Technol 27(10):1109-1123

Huygen HH (1976). Laboratory steamfloods in half of a five-spot. In: SPE annual fall technical conference and exhibition. society of petroleum engineers

Huygen HH, Lowry WE Jr (1983) Steamflooding Wabasca tar sand through the bottom water zone-scaled model tests. Soc Petrol Eng J 23(01):92-98

Jadhawar PS (2010) $\mathrm{Co}_{2}$-assisted gravity drainage EOR: numerical simulation and scaling models study (Doctoral dissertation).

Kimber KD, Ali SM, Puttagunta VR (1988) New scaling criteria and their relative merits for steam recovery experiments. J Can Petrol Technol 27(04)

Langhaar HL (1951) Dimensional analysis and theory of models, vol 2. Wiley, New York

Leverett MC, Lewis WB, True ME (1942) Dimensional-model studies of oil-field behavior. Trans AIME 146(01):175-193

Lo HY (1977) Laboratory model study of steamflood oil recovery. Petroleum Recovery Institute, IR-5

Mattax CC, Kyte JR (1962) Imbibition oil recovery from fractured, water-drive reservoir. Soc Petrol Eng J 2(02):177-184

Murphy G (1950) Similitude in engineering. Ronald Press Co., New York

Nielsen RL, Tek MR (1963) Evaluation of scale-up laws for two-phase flow through porous media. Soc Pet Eng J 3(02):164-176. https:// doi.org/10.2118/494-PA

Novakovic D (2002) Numerical reservoir characterization using dimensionless scale numbers with application in upscaling. Doctoral dissertation, Louisiana State University 
Poettmann FH, Hocott CR, Bond DC (1974) Secondary and tertiary oil recovery processes. Interstate Oil Compact Commission, Oklahoma City

Pujol L, Boberg TC (1972) Scaling accuracy of laboratory steam flooding models. In SPE California regional meeting. Society of Petroleum Engineers

Pursley SA (1974) Experimental studies of thermal recovery processes. In Heavy oil symposium, Maracaibo

Rahman A (2018) Development of scaling criteria and numerical simulation study of steam flooding process (Master's thesis, Memorial University of Newfoundland)

Rahman A, Happy FA, Ahmed S, Hossain ME (2017) Development of scaling criteria for enhanced oil recovery: a review. J Pet Sci Eng 158:66-79

Rapoport LA (1955) Scaling laws for use in design and operation of water-oil flow models. Trans AIME 204:143

Rapoport LA, Leas WJ (1953) Properties of linear waterfloods. J Pet Technol 5(05):139-148. https://doi.org/10.2118/213-G

Rojas GA (1985) Scaled model studies of immiscible carbon dioxide displacement of heavy oil

Ruark AE (1935) Inspectional analysis: a method which supplements dimensional analysis. J Mitchell Soc 51:127-133
Seve BJ, Pottier J (1963) French engineers review soviet modeling practices. J Pet Technol 15(03):581-588. https://doi. org/10.2118/532-PA

Shook M, Li D, Lake LW (1992) Scaling immiscible flow through permeable media by inspectional analysis. In Situ New York 16:311

Singhal AK (1980) Physical model study of inverted seven-spot steamfloods in a pool containing conventional heavy oil. J Can Pet Technol 19(03):123-134

Sonin AA (2001) The physical basis of dimensional analysis. Department of Mechanical Engineering, MIT, Cambridge, p 57

Stegemeier GL, Laumbach DD, Volek CW (1980) Representing steam processes with vacuum models. Soc Pet Eng J 20(03):151-174

Van Meurs P (1956) The use of transparent three-dimensional models for studying the mechanism of flow processes in oil reservoirs. In SPE-678-G, paper presented at petroleum branch fall meeting in Los Angeles

Yortsos YC (1979) Analytical modelling of oil recovery by steam injection (Doctoral dissertation, California Institute of Technology)

Publisher's Note Springer Nature remains neutral with regard to jurisdictional claims in published maps and institutional affiliations. 